\title{
REVIEW OF PIPELINE SPAN ANALYSIS
}

\author{
Abdulhakim Adeoye Shittu ${ }^{1,2, *}$, Kara Fuat $^{1}$, Ahmed Aliyu $^{3}$, Obinna Unaeze ${ }^{1}$ \\ ${ }^{1}$ Offshore Energy Engineering Centre, School of Water, Energy and Environment, Cranfield \\ University, Cranfield, Bedfordshire, United Kingdom, ${ }^{2}$ Department of Mathematics and \\ Statistics, Federal University Wukari, Taraba State, Nigeria, ${ }^{3}$ Department of Chemical \\ Engineering, Faculty of Engineering, Federal University Wukari, Taraba State, Nigeria. \\ *Corresponding Author email: hakimabdu105@yahoo.co.uk, a.a.shittu@cranfield.ac.uk
}

\section{ABSTRACT}

This paper mainly review the state-of-the-art developments in the field of hydrodynamics of offshore pipelines, identifying key tools for analysis of pipeline free spans, their applications, their qualifying characteristics and capabilities and limitations. These different analytical, numerical, and semi-empirical tools available for predicting such hydrodynamic loads and its effects include VIVANA, PIPESIN, VIVSIM, SIMULATOR, FATFREE among others. Inherent in these models are current effects, wave effects and/ or pipe-soil interactions. Amongst these models, the most attention was given to the new VIVANA model since this model take into account the vortex-induced effects with respect to free spanning Pipelines (which have dominant effect in the span analysis in deep water) better than other semiempirical models (such as Shear 7). Recent improvements in VIVANA include its ability to have arbitrary variation in speed and direction of current as well as ability for calculation of pure IL and combined IL-CF response. Improvements in fatigue assessments at free spans i.e pipe-soil interaction has been achieved through the combined frequency domain and non-linear time domain analysis methodology adopted. Semi-empirical models are still the de-facto currently used in the design of free spanning pipelines. However, there is need for further research on free span hydrodynamic coefficients and on how in-line and cross-flow vibrations interact. Again, there is still the challenge due to VIV complexity in fully understanding the 
fluid structure interaction problem as there is no consolidated procedure for its analysis. It has been observed that there is large scatter between the different codes adopted in the prediction of fatigue damage as there lacks full scale test data devoted to determination/validation of the coefficients used in the semi-empirical models. A case study of the preliminary design of a typical 48 inches Pipeline has been presented in this work to demonstrate the use of the free span analysis tool, DNV RP F105. Excel spreadsheet has been applied in the execution of formulars. This review paper is the first of its kind to study the state-of- the-arts development in pipeline free span analysis models and demonstrate the use of analysis tool, DNV for MAFSL calculation. Hence, information obtained from this paper would be invaluable in assisting designers both in the industry and Academia.

Keywords: Pipeline spans; Vortex Induced Vibration; Mathematical models; Hydrodynamic loads; MAFSL

\section{Introduction}

Anfinsen (Afiinsen, 1995) made an illustration of how parameters such as Hydrodynamic forces, support conditions, height of free span, span configuration, damping, analysis models and axial force in pipeline influence free span calculations. Also the developments within free span calculations since the petroleum industry and field developments started on the Norwegian continental shelf were presented. Tura and Vitali (Tura \& Vitali, 1991) conducted a series of tests to serve as basis for generating a mathematical model. The main objective was to measure the response of a pipeline to varying intensities of steady currents. It was claimed that the nonlinear behaviour of free spanning pipelines exposed to steady currents has a strong influence on the onset of hydroelastic synchronization due to vortex shedding; the mathematical model revealed the existence of a geometrical condition needed for the onset of synchronization for long free spans and can be used successfully for predictive purposes when applied to real free spans to assess several key factors, among other results. Kaye, et al. (Kaye 
et al., 1993), described a stringent methodology for the assessment and rectification of pipeline free spans with respect to a certain field. This methodology comprised of two parts each with two stages, and consist of preliminary stress and vibration frequency checks succeeded by comprehensive strain and fatigue life checks where appropriate; also discussed was the use of an ROV based freespan rectification technique, among others. Jones (Jones, 1995), studied the precision of quasi-static methods of analysis for the prediction of the inelastic behaviour of structural impact issues and made comparison with experimental result in most cases. It was claimed that quasi-static analyses were much effortless than full dynamic plastic analyses for the velocity range studied, among others. Park and Kim (Park \& Kim, 1997), analysed static and dynamic free spans in order to determine the allowable free span length and studied the variation of the allowable lengths at specialised boundary conditions (BCs). It was claimed that non dimensional curves were developed to determine easily the exact allowable lengths for subsea pipelines. Kapuria et al. (Kapuria, Salpekar, \& Sengupta, 1999), presented an analytical solution for fatigue response resulting from free spanning submarine pipeline cross flow vibration on semi-infinite elastic soil beds supports at the ends. It was claimed that the fatigue life decreases as axial compression increases or tension decreases in certain conditions, the onset of cross flow vibration criteria may yield highly conservative value for allowable free span in some cases, etc.

Mork and fyrileiv (Mørk, Fyrileiv, Nes, \& Sortland, 1999), proposed an approach for the evaluation of non-stationary free spans since the assessment of such spans are more complex and implies consideration of temporal factors such as quasi-stationary span conditions, etc. it was claimed that for pipelines with moderate free span development fatigue acceptance criteria and an intervention strategy may be based on certain span lengths such that the criteria are normally adequate and provide firm decision criteria for most spans identified during the survey, etc. Reid, et al. (Reid, Grytten, \& Nystrom, 2000) provided interpretation of some 
methods in hydrodynamic fatigue assessment for free spanning pipelines. A three dimensional (3D) finite element (FE) method was adopted for the Eigen-value analysis and a high speed numerical tool incorporating wave loading and VIV was used for the fatigue calculations. It was claimed that parameters such as damping, water depth, seabed gap, etc. have an effect on the fatigue damage both in the cross flow and in-line directions. Choi (Choi, 2001), developed a stringent procedure for free spanning analysis of pipelines. It was claimed that the result of a study revealed that axial load influences the natural frequencies (applying the energy balance equation with solutions of beam-column equations) and allowable span lengths of the pipeline for various BCs, etc. Chen and Cheng (Chen \& Cheng, 2002), simulated a 3D flow near a free spanned pipeline using a fractional step FE method. The study revealed that a spiral vortex tube was formed around and extends around the span shoulder which conforms to existing literature, and that there are substantial shear stress concentrations in the span shoulder area. Nielsen, et al. (Nielsen, Søreide, \& Kvarme, n.d.), studied the VIV response of long free spanning pipelines in current. They claimed that by increasing the span length several mode shapes could be excited and that due to the sag effect characterised by long free spans, dynamic properties in the horizontal and vertical direction are dissimilar, the VIV response as observed from model tests as well as hypothesis responsible for this was discussed, among others. Fyrileiv and Collberg (Fyrileiv \& Collberg, 2005), discussed the influence of pressure in pipeline design (effective axial force) in general and in the DNV codes. Several discussions were made some of which include the following: Effective axial force concept is simple and accounts for pressure effects efficiently; the effective axial force expression in the DNV code is correct although simplified; the hoop stress and true wall force have an influence on the local buckling; natural frequency decreases as the internal pressure increases, etc. Eigbe, et al. (Eigbe, Fletcher, Hensley, Ling, \& Routh, 2006), performed free span remediation studies for a deepwater flowline system consisting of High temperature/ High pressure Pipe in pipe (HT/ HP PIP) 
flowlines in the Gulf of Mexico traversing rugged seabed terrain which consists of an escarpment along the route selected. The result of the preliminary analysis shows that seabed intervention with the use of engineered supports was necessary at some of the spans. Prevention of excessive bending as well as the assessment of span support, overburden impact with respect to structural integrity was key emphasis. ANSYS FE modelling of as laid flowlines was included in the study. It was claimed that a fully vetted and field-proven HT/ HP PIP analysis tool available for similar applications was produced.

This review aims to present the state-of-the-art developments in the field of hydrodynamics of offshore pipeline, identifying key models for analysis of pipeline free spans, their applications, their qualifying characteristics and/ or capabilities and limitations. In order to identify relevant sources, a systematic review approach has been followed, focusing search mainly on journals, conference papers and industry documents which has been published using predefined key words on Science direct, One petro, Scopus, ASME digital collection, Company websites, among others. Also industry leaders in the design for pipeline free spans were contacted for details of experience with existing Pipeline free span analysis models.

Pipeline span analysis is an important tool used in offshore and ocean technology for structural failure due to overstress from steady state loads, fatigue failure as a result of vibrations from dynamic loads (such as Vortex Induced Vibrations, VIV) and severe damage due to third party activities (hooking from trawl gears or drop objects) (Palmer, A. \& King, A., 2004; Shittu, 2012; Xing, 2011). According to Gou et al. (Guo, Song, Ghalambor, Lin, \& Chacko, 2005), pipeline spanning usually occurs when the contact between the pipeline and seabed is lost over a long depression on a rough seabed. Present research in the oil and gas industries are moving towards harsher environments often characterized by uneven seabed and deep water (Ai \& Sun, 2009). The number of submarine pipelines being laid in such environments is increasing at a massive rate in different parts of the world. Thus, free spanning pipelines are becoming more 
frequent and are often unavoidable during pipeline installation (Project consulting service inc., 1997).

The formation of submarine pipeline spans may have a critical influence on the safety and integrity of the pipelines (Jp \& Partners, 1993; Shittu, 2012). Recent reports have shown failures due to free spanning of pipelines thus necessitating an increased attention on pipeline span analysis. Spans can be developed during pipelay as a result of irregularity in the bedform (coupled with factors such as pipe weight, pipe stiffness, among other), service life of the pipeline, the dynamic seabed-scouring and horizontal movements of the seafloor (Rezazadeh, Zhu, Bai, \& Zhang, 2010). In order to reduce costs and provide more reliable pipelines on extremely rugged seafloor, there is a need to understand VIV and thus improve methods, existing computer programs and guidelines needed for design verification.

VIV, is a major source of dynamic stresses in free span pipelines as a result of steady current. If the vortex shedding frequency which is caused by normal flow reaches to the natural frequency of pipeline, pipeline starts to vibrate and VIV occurs which may cause pipeline fatigue damage (Shabani, Taheri, \& Daghigh, 2017). Deep water pipelines are highly susceptible to this effect since wave induced velocities and accelerations will decay with increasing water depth (Koushan, 2009). There are several models used for the prediction of VIV of free span pipelines, some of which are empirical models. Most empirical models are based on frequency domain dynamic solutions and linear structural models (Larsen, Koushan, \& Passano, 2002). However, important non-linearities are inherent in free span pipelines that should be accounted for. Both tension variation and pipe-seafloor interaction impacts on nonlinear behaviour, which entails most empirical models, will have significant limitations when dealing with the free span case. Therefore, the need for time domain methods is thus apparent. 
There are several models for prediction of hydrodynamics in wave dominated waters (at shallow water depths) such as the Morison's model and the wake models. Theoretical background on the Morison's model can be found in (B. M. Sumer \& Fredsoe, 1997) while on wake models can be found in (Aristodemo, Tomasicchio, \& Veltri, 2011; Ruby \& Hartvig, 2008; Sabag, Edge, \& Soedigdo, 2000; Soedigdo, Lambrakos, \& Edge, 1998). Also, the theoretical background on wave theories and there application can be found in (Sarpkaya \& Isaacson, 1981). Further, the theoretical background on Vortex Shedding phenomenon can be found in (Choi, 2001; Jp \& Partners, 1993; Raven, Stuart, Bray, \& Littlejohns, 1985; Sarpkaya \& Isaacson, 1981; B. M. Sumer \& Fredsoe, 1997). The theoretical background on fatigue phenomenon can be found in (Det Norske Veritas, 2000, 2007; Rippon, Shah, \& White, 1986; Ruby \& Hartvig, 2008). Relevant references on span creation mechanisms include (Det Norske Veritas, 2007; Shittu, 2012; Shittu \& Kara, 2018; Wang, Banneyake, Huang, Jukes, \& Eltaher, 2011; Wei, Lihua, Guangxue, \& Rongmin, 2010). Span creation mechanisms due to environment include scouring, sandwaves, liquefaction, underwater landslides, etc. Several studies carried out under scouring phenomenon includes (Alam \& Cheng, 2010; Cao \& Qin, 2010; Chen \& Cheng, 2002; L Cheng, Zang, Zhao, \& Teng, 2008; Liang Cheng, Yeow, Zhang, \& Teng, 2009; Liang Cheng \& Zhao, 2010; Etemad-Shahidi, Yasa, \& Kazeminezhad, 2011; Fard, Yeganeh-Bakhtiary, Cheng, \& Khayyer, n.d.; Gao, Yang, Yan, \& Wu, 2006; Huai, Wang, Qian, \& Han, 2011; Liang \& Cheng, 2005; Liang, Cheng, \& Li, 2005; Liang, Cheng, \& Yeow, 2005; Lu, Li, \& Qin, 2005; Mirmohammadi \& Ketabdari, 2011; Myrhaug, Ong, Føien, Gjengedal, \& Leira, 2009; Myrhaug, Ong, \& Gjengedal, 2008; Smith, 2007; M. Sumer \& Fredsoe, 2002; Wu \& Chiew, 2011; Yang, Shi, Han, Wu, \& Sun, 2010; Yeganeh-Bakhtiary, Kazeminezhad, Etemad-Shahidi, Baas, \& Cheng, 2011; Zang, Cheng, Zhao, Liang, \& Teng, 2009; Zhao \& Cheng, 2010) and under sandwaves include (da Silva, Temperville, \& Seabra Santos, 2006; Davies, Van Rijn, Damgaard, Van de Graaff, \& Ribberink, 2002; Jiang \& Lin, 
2010; N.L. Komarova \& Newell, 2000; Natalia L. Komarova \& Hulscher, 2000; Li, Lin, Jiang, \& Fan, 2011; Martel, 2004; Nemeth, 2003; A. A. Németh, Hulscher, \& Van Damme, 2006, 2007; Attila A. Németh, Hulscher, \& De Vriend, 2002; Nodine et al., 2007; Tonnon, van Rijn, \& Walstra, 2007; Van den Berg, 2007; van den Berg, Sterlini, Hulscher, \& van Damme, 2012; van Santen, de Swart, \& van Dijk, 2011).

Excessive conservatism overestimates the threat to pipeline integrity, and leads to unnecessary capital and operating expenditure in free span control and intervention work (Esplin \& Stappenbelt, 2011). This is inherent in the approximate response models in the DNV design guidelines. However, there are alternative methods available to overcome these limitations according to the DNV RP F105 (Det Norske Veritas, 2006) recommendations.

Based on interaction of adjacent spans free span analysis is performed in two ways; static analysis (for isolated span i.e. single span) and dynamic (for interacting span i.e. multi span). The procedure for determining the fatigue life capacity of a pipeline are thus: the DNV classified the free spanning pipeline behaviour into three categories based on ratio of span length to pipeline diameter: beam dominant behaviour (for $30<\mathrm{L} / \mathrm{D}<100$ ), combined beam and cable behaviour (for $100<\mathrm{L} / \mathrm{D}<200$ ) and cable dominant behaviour (for $\mathrm{L} / \mathrm{D}>200$ ) (Shabani et al., 2017). In the first category, pipeline response can be estimated by deterministic theories i.e. Bernoulli's beam theory. However, in the second and third class, the beam theory is not applicable and the dynamic response must be predicted by solving differential equation i.e. equation of motion (Shabani et al., 2017).

The importance of the consideration of pipe-soil interaction in span analysis cannot be overemphasized. The soil stiffness in both horizontal and vertical directions affects the maximum amplitude response of oscillation.

This paper is structured as follows. Section 2 discusses models for the prediction of behaviours of pipeline within a given hydrodynamic environment presenting different analytical tools 
currently used and current advancement in the Computational Fluid Dynamics (CFD) as well as Finite Element Analysis (FEA) methods. Section 3 presents the methods for model prediction discussing the current most common tools used in the industry for Vortex Induced Vibration, Pipe-soil interaction, fatigue characteristics and response prediction among other phenomena. Section 4 discusses the comparison between models used for predicting the VIV as well as FEA phenomena which are design factors of critical importance for the structural response in Pipeline span analysis in deep water. Section 5 presents a case study where design of a typical 48 inches pipeline against free spans is performed in order to demonstrate pipeline free span analysis according to DNV. Section 6 and 7 presents the recommendations and conclusions respectively.

\section{Models for prediction of behaviours of pipeline in a given hydrodynamic environment.}

\subsection{Simulator ${ }^{\mathrm{TM}}$}

Simulator can be used for complex marine operations such as 3D analysis of pipelaying including free spans with use of SIMLA, etc. Simulator provides free span analysis tools for interdisciplinary teamwork, ROV positioning, etc. during pipe laying, lifting operations, etc. but irregular seabed topography coupled with large water depths and strong ocean currents, dynamics of vessels and module, etc. pose challenges.

\subsection{PIPESIN ${ }^{\mathrm{TM}}$}

PIPESIN is a three-dimensional numerical model which can be applied to simulate interactions between a pipeline and dynamic seabed. It can be used to assess free span development and calculate the maximum potential free span length and related duration. PIPESIN is suitable to simulate interactions of a pipeline and migrating sandwaves. It is also possible to apply PIPESIN to an existing pipeline on seabed (length typically of 500 to $1000 \mathrm{~m}$ ) with arbitrary configuration. Furthermore PIPESIN can be used to assess feasibility of use of a spoiler to 
increase self-lowering and improve pipeline stability. PIPESIN can model processes such as initial pipeline lowering, nearbed wave and current field, onset of scour, etc.

\subsection{VIVSIMTM $^{\mathrm{TM}}$}

VIVSIM is JP Kenny's software tool for fatigue assessment of wave and VIV of free spanning pipelines. The program has been established in accordance to the DNV RP-F105, "Free Spanning Pipelines". VIVSIM is based on FORTRAN and seamlessly integrated with JP Kenny Norge's Pipeline Simulator tool. It also has compatibility with any FE models applicable for in-place analyses as long as the input is on a VIVSIM compatible format.

\subsection{Models for span analysis during installation}

\subsubsection{SAGE Profile 3D ${ }^{\mathrm{TM}}$}

SAGE Profile 3D was established by Fugro GeoConsulting Belgium (FGBC). This was specifically designed to help pipeline engineers with their designs. It provides excellent basis providing rapid efficient pipeline simulations for tasks such as static free span evaluation, pipeline route optimization, upheaval/ lateral buckling, etc. This new computational engine allows full 3D pipeline stress analyses with capability of handling large deformation issues such as lateral buckling and accurate modelisation of the pipe-soil interaction by the use of advanced plastic soil models. Simulation results can be cross checked against various design codes such as the DNV, ASME B31.8, etc. These cross checks can be selected and conducted as part of the post processing.

The SAGE Profile 3D (SP3D) includes the SP3D Interface and the SimPipe 3D FE engine. The SP3D Interface comprises the Editor module, the Analysis module and the Viewer module. Post-processor functions include the code check and the span check.

In areas where the pipe passes over a sand wave or spans a depression, SAGE Profile 3D can take into consideration pipe settlement into the span shoulders. The plastic soil models provide a realistic indication of pipe embedment in all load cases. 


\subsubsection{OFFPIPE ${ }^{\mathrm{TM}}$}

OFFPIPE is a proprietary computer program developed by Robert C. Malahy for the analysis of structural problems encountered during the installation of offshore pipelines. This program also conducts the free span analyses during installation some of which are described thus: Nonlinear (both geometric and material) modelling of two and three dimensional pipe spans on the ocean floor. The seafloor is modelled as a continuous elastic-plastic foundation using an irregular, two or three dimensional seabed surface profile with any desired roughness. Environmental loadings can include steady currents, regular waves and wave spectra, and vortex shedding. Other loadings can include residual tension, internal fluid contents, internal/external pressure, and thermal expansion due to fluid temperature. Loadings can be applied and analyses can be conducted sequentially. The history of pipe displacements (soil friction) and deformations (plasticity) can be tracked to permit the results of the installation and hydrostatic test to be used as initial conditions in subsequent analyses of service loadings such as cyclic thermal expansion, etc.

In static analyses, OFFPIPE calculates the pipe stresses and deformed geometry at every point along the pipeline and identifies all points at which pipe spans exist while in dynamic analyses, OFFPIPE calculates the natural frequencies of pipe spans, and determines the pipe stresses and displacements resulting from wave loadings and vortex shedding, etc.

\subsection{Other Mathematical Models}

Yeganeh et al. (Yeganeh Bakhtiary, Ghaheri, \& Valipour, n.d.), described how the pattern of current induced drag force can be obtained using a numerical model constructed based on the Reynolds Averaged Navier-Stokes (RANS) equations in combination with a standard $k-\varepsilon$ turbulence model for turbulence closure for incompressible viscous flow in two dimensional Cartesian coordinate system. The main equations are

(1) to (8). 


$$
\begin{aligned}
& \frac{\partial U}{\partial x}+\frac{\partial V}{\partial y}=0 \\
& \frac{\partial U}{\partial t}+U \frac{\partial U}{\partial x}+V \frac{\partial U}{\partial y}=-\frac{1}{\rho} \frac{\partial p}{\partial x}+\frac{\partial}{\partial x}\left[2 \Gamma \frac{\partial U}{\partial x}\right]+\frac{\partial}{\partial y}\left[\Gamma\left(\frac{\partial U}{\partial y}+\frac{\partial V}{\partial x}\right)\right] \\
& \frac{\partial V}{\partial t}+U \frac{\partial V}{\partial x}+V \frac{\partial V}{\partial y}=-\frac{1}{\rho} \frac{\partial p}{\partial y}+\frac{\partial}{\partial y}\left[2 \Gamma \frac{\partial V}{\partial y}\right]+\frac{\partial}{\partial x}\left[\Gamma\left(\frac{\partial U}{\partial y}+\frac{\partial V}{\partial x}\right)\right] \\
& \Gamma=\mathrm{v}+v_{t} \\
& \frac{\partial k}{\partial t}+U \frac{\partial k}{\partial x}+V \frac{\partial k}{\partial y} \\
& =\frac{\partial}{\partial x}\left[\left(v+\frac{v_{t}}{\sigma_{k}}\right) \frac{\partial k}{\partial x}\right]+\frac{\partial}{\partial y}\left[\left(v+\frac{v_{t}}{\sigma_{k}}\right) \frac{\partial k}{\partial y}\right]+P_{r}-\varepsilon \\
& \frac{\partial \varepsilon}{\partial t}+U \frac{\partial \varepsilon}{\partial x}+V \frac{\partial \varepsilon}{\partial y} \\
& =\frac{\partial}{\partial x}\left[\left(v+\frac{v_{t}}{\sigma_{\varepsilon}}\right) \frac{\partial \varepsilon}{\partial x}\right]+\frac{\partial}{\partial y}\left[\left(v+\frac{v_{t}}{\sigma_{\varepsilon}}\right) \frac{\partial \varepsilon}{\partial y}\right] \\
& +\frac{\varepsilon}{k}\left(C_{1 \varepsilon} P_{r}-C_{2 \varepsilon} \varepsilon\right) \\
& P_{r}=v_{t}\left[2\left(\frac{\partial U}{\partial x}\right)^{2}+2\left(\frac{\partial V}{\partial y}\right)^{2}+\left(\frac{\partial U}{\partial x}+\frac{\partial V}{\partial y}\right)^{2}\right] \\
& v_{t}=\frac{C_{\mu} k^{2}}{\varepsilon}
\end{aligned}
$$

Valipour et al. (Valipour, Bakhtiary, Ghaheri, \& Kazeminezhad, 2008), developed a finite element method (FEM) model by using the ANSYS software in order to solve the fundamental equations of motion of pipe. In the software used, the pipe 16 element was selected to discrete the pipe at the free-span section into the number of elements. Then the calculated cyclic loads became the inputs of the model on supports.

The wave profile was simulated using Non-deterministic Spectral Amplitude in Gaussian sea state and using linear wave formulation to calculate wave-induced forces. 
The way in which the vortex shedding frequency (VSF) leads to a sinusoidal pattern in the net acting drag forces was depicted. The acting drag force can be expressed as eq.(9).

$$
f(z, t)=f_{D}\left(1+A_{c} \cos (V S F \times t)\right)
$$

Huang and Xu (X. G. Huang \& Xu, 2010) developed a dynamic differential equation of subsea pipeline spans based on the Hamilton principle. A constraint-equivalent method was used to address the pipeline span boundary conditions on the linear elastic seabed. Internal flow velocity and seabed stiffness's influence on the pipeline's lateral deformation and bending stress were studied by the static analysis, while the initial relationships between the internal flow velocity and the foundation stiffness to the natural frequency of pipeline span were examined by the dynamic analysis among other studies.

It was found that the lateral deformation increases with the increment of internal flow velocity, but decreases with the increment of seabed stiffness, etc. It was claimed that numerical examples show that the proposed procedure is feasible and can address the seabed support in a more accurate way, which can break away from the two traditional models: simply supported model and fixed-fixed model. The effect of the internal flow and seabed stiffness on the mechanical behaviour of pipeline span was also claimed to be clarified.

Ai and Sun (Ai \& Sun, 2009), investigated the effect of internal flow velocity and functional loads on VIV response. This involved the derivation of a differential equation based on the Hamilton's principle for the definition of a fluid conveying pinned-pinned tensioned spanning pipeline; the VIV response being calculated according to DNV RP F105 under different functional loads. It was claimed that based on the result obtained, the pipeline natural frequency increases with decreasing internal flow speed/ compression. Tension increase can also result in higher frequencies, etc. 
The author claims that the research may be used as a reference for the study of sensitivity of functional loads (tension and compression force, etc.) on the allowable free span length and also provide crucial information pertaining subsea pipeline design.

Pontaza et al. (Pontaza et al., 2010), studied the fluid structure interaction simulations of a pipeline span exposed to sea bottom currents in order to gain an insight into VIV of pipeline spans near seafloor such that such insight can be manifested into sets of free spanning pipelines' design guidelines in the long run. The pipeline span's VIV response was predicted by a 3D viscous incompressible Navier-stokes solver - beam finite element solver coupling. Several parameters including flow turbulence, seabed proximity, submerged weight influenced pipeline sagging, etc. were considered in the FSI simulation.

Esplin and Stappenbelt (Esplin \& Stappenbelt, 2011), presented the probabilistic analysis (Monte Carlo approach based on the DNV RP F105 (Det Norske Veritas, 2006) recommendation) of a typical free spanning pipeline within the Norwegian deepwater development area in order to address the excessive conservatism inherent in the deterministic approach DNV RP F105. It was claimed that the deterministic treatment of a bilinear S-N fatigue curve which retains the conservatism inherent in the experimental data representation discerns this probabilistic analysis from those hitherto undertaken, etc. It was claimed that the study produced evidence consistent with the proposition that traditional free span assessment are overly conservative, etc. It was claimed also that this methodology has the potential with respect to prevention of free span intervention and significant associated costs in situation that would otherwise proceed in the conventional deterministic form of assessment. 
Elsayed, et al. (Elsayed, Fahmy, \& Samir, 2016) presented an approach for screening subsea pipelines against free spanning. The approach is based on the use of non-linear finite element model. The FE model constructed using the FE package ANSYS was used to compute the combined stresses/ lateral displacement acting on offshore pipelines due to combined hydrodynamic loads including wave/ current effects. In ANSYS, the pipe-soil interaction, and environment can be modelled and as such friction forces and soil stiffness can be incorporated in the analysis. Based on the results obtained It was claimed that the proposed approach can be a valuable tool for pipeline designers for assessment of pipeline free spans.

Drago et al. (Drago, Pigliapoco, \& Ciuffardi, 2007), developed the PIPESOIL and SPAFAT (established based on the DNV RP F105 (Det Norske Veritas, 2006)) such that it can be coupled and used to assess the fatigue damage accumulation (due to the influence of VIV and/ or waves ) of an evolving free span (i.e. incorporating mechanisms defining pipe-soil configuration such as scouring) under forcing agents. The PIPESOIL model incorporates isolated free span morphology and it is based on semi empirical relationships defining tunnelling and onset of scouring, pipe self-lowering, free span formation and leeside erosion.

The SPAFAT program permits assessment of both isolated and interacting spans either in single or multiple mode vibration. The general analysis approach steps include: Eigen value analysis which gives natural frequencies and corresponding modal shapes for vibration of the free span due to drag and lift forces, using force/ response models for analysis to achieve the stress ranges from environmental actions.

Based on the results obtained it was claimed that since the use of a large number of wave and current time series produced a statistically significant sample to evaluate the probability of occurrence of unacceptable fatigue damage for different pipe and water depth scenarios, a 
conclusion is drawn that the coupled models are useful tools to select upon which are the better corrective actions to adopt and their extent.

\section{Methods for Model Prediction}

An illustration showing the most common VIV prediction models is presented in Figure 1.

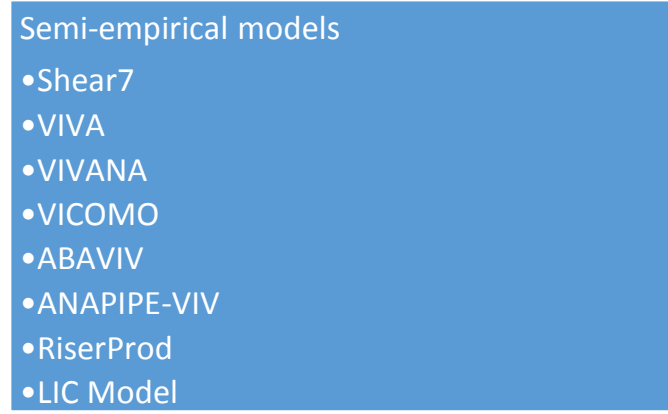

Computational Fluid Dynamics (CFD)

- Norsk Hydro

- USP

- Deepflow

- VIVIC

\section{Orcina Orcaflex \\ - Vortex Tracking \\ - Wake oscillator}

Figure 1: Some VIV prediction Models

\subsection{VIVANA ${ }^{\mathrm{TM}}$}

According to Passano et al. (Passano, Larsen, \& Wu, 2010), VIVANA was originally developed by MARINTEK and the Norwegian university of Science and Technology (NTNU) to predict cross flow response due to VIV. The fluid-structure interaction in VIVANA is described using added mass, excitation and damping coefficients. Default curves are available or the user may input other data.

VIVANA (Larsen et al., 2002) is a semi-empirical frequency domain program based on a 3D finite element formulation of the structure (by applying analysis software, RIFLEX) and a VIV response analysis model. 
VIVANA originally could handle only cross flow excitation but pure in-line excitation was later added. Recently, simultaneous cross-flow and in-line excitation has also been included. At present the excitation in the cross-flow and in-line direction is not coupled. Coefficients for simultaneous cross flow and in-line excitations were proposed and are available in VIVANA. VIVANA incorporates a set of lift coefficient curves where each curve is described by three points. The points are given as functions of the non-dimensional frequencies.

The response calculations are performed at discrete response frequencies. The main VIV coefficients are the CF and IL added mass and excitation coefficients. The default VIV coefficients included in the program could be applied or the user could specify other coefficients. Hydrodynamic damping is used outside the excitation zone.

The structure is modelled by applying the finite element method incorporated with beam and cable elements. The program also may include varying cross-sectional properties such as diameter, stiffness and VIV coefficients. Influences of seafloor contact are represented as linear springs. The current profile may vary in direction and/ or be sheared.

The assumption that the response is occurring at one or more discrete eigen frequencies is central to the program. The mode related is utilised as the initial estimate of the response and response iterations performed at each frequency until consistency is achieved between the response and the VIV loads. The analysis consists of the following main steps:

3.1.1 Compute the eigenfrequencies which are possible response frequencies. Since the added mass depends on the non-dimensional frequency, iterations are conducted for each response frequency to make sure the resulting eigen frequency are consistent.

3.1.2 Computation of response at each response frequency, the response frequency is kept constant and iterations are performed until the response and the excitation are consistent. 
3.1.3 Computation of resulting response stresses and fatigue damage from all response frequencies. Competing frequencies could either appear concurrently or consecutively (time sharing).

VIV loads may be applied in the local IL direction, local CF direction or in both directions. Several strategies are adopted in order to determine the eigen frequencies that are possible response frequencies in the three cases. In the case of pure CF loading, the added mass coefficients are dependent on the non-dimensional frequency. The added mass in the CF direction is adjusted for each eigen frequency until the eigen frequency and the added mass are consistent.

In the case of combined CF and IL loading the current strategy is to find the possible CF response frequencies first and then adjust the IL added mass to obtain an IL eigenfrequency that is two times the CF frequency. Depending on the values of the still water IL eigenfrequencies near the target frequency, the IL added mass is shifted up or down in order to get an eigen frequency with the target value. The added mass is reduced and a lower eigen frequency increased if the target frequency is in the lower quarter of an interval. Otherwise, the added mass is increased and a higher eigen frequency is decreased. The program will therefore tend to give IL response at higher of the two possible mode shapes. This is assumed to be conservative as a higher mode will tend to give higher curvature, bending stress and fatigue damage.

Different sets of the IL excitation coefficients are used for pure IL loading and for combined CF and IL loading. This is in agreement with the experience that IL response increases after the onset of CF response.

In the present version of the program, there is no interaction between the response calculations at the CF and IL frequencies. This interaction may be included in the response iterations in 
VIVANA when data for the excitation coefficients as a function of the response at both frequencies becomes available.

Larsen et al. (Larsen et al., 2002), presented an approach based on the combination of an empirical linear frequency domain model, and a non-linear time domain structural model. It was claimed that the advantage of using this model is that stresses at the shoulders are more precisely defined which is crucial as fatigue damage in many situations will be largest in such areas. It was noted that the pipe-seafloor interaction is crucial for accurate stress prediction, and that a non-linear time domain model will provide the most precise result. Based on analysis results, it was claimed that the model predicts on-set of cross flow VIV and the transition from one dominating mode to another reasonably well. However, verification by means of experiments and further work on the inline-cross flow response interaction was recommended among other recommendations.

Passano et al. (Passano et al., 2010), compared the predictions of VIV from a semi-empirical program (VIVANA) to experimental data. The data was obtained from a VIV model test program of a pipeline on free span by applying a long elastic pipe model. Comparisons between inline and cross-flow vibrations were made.

The data compared were for two models denoted as Model case 10 and 42 having $L=11.413$, $D=0.0326$, Bending stiffness $E I=0.203$, etc. and Model case 75 having the same length and bending stiffness but $D=0.03504$, etc. Experiments were conducted for the first two series (i.e. 10 and 42) using the phase I/II model, while the last was with the phase III model which represents different scenarios/ conditions. In this case study, under certain conditions such as flow velocities, etc. the analyses were performed the IL VIV loads only as well as with the combined CF and IL VIV loads option. 
A typical example analysis in the case study, the test series 10 - analyses shows that the pure IL loading is valid only up to the current level where CF response commences.

The CF loading commenced at a current velocity of $0.06 \mathrm{~m} / \mathrm{s}$ and the analysis with CF and IL loading began with response frequencies corresponding to CF mode 1 and IL loading start with response frequencies corresponding to CF mode 1 and IL mode 2, shift to CF 1 and IL 3, CF 2 and IL 3, CF 2 and IL 4, CF 3 and IL 4 and finally CF 3 and IL 5.

It was observed that the IL response frequency, corresponding mode and maximum response from the first analysis with both CF and IL loading are in good agreement with the values from the analysis with only IL loading. As expected, the analysis with only IL loading yields less IL response than combined $\mathrm{CF}$ and IL loading at higher current levels.

For the CF response frequencies and the response frequencies for pure IL loading, the added mass is dependent on the non-dimensional frequency and is hence consistent with the response frequency. The added mass for the IL response frequency for combined loading is the value required to offer an IL eigen frequency at twice the CF response frequency.

At $0.04 \mathrm{~m} / \mathrm{s}$ there is only IL loading resulting in a small IL first mode response and no CF response. At $0.10 \mathrm{~m} / \mathrm{s}$ there is a large CF first mode response and a significant IL third mode response.

It was claimed that good predictions of IL response up to and beyond the start of CF response for the three test series in their study were achieved for the analyses with pure IL loading. The analyses with combined CF and IL loading produced satisfactory response predictions for test series 10 . This test series was characterised by long span and IL modes 2 - 4 were excited in the experiments with CF response. Satisfactory response predictions were also obtained for the short span experiments with CF mode 1 and IL mode 2; the two tests in test series 42 above $0.4 \mathrm{~m} / \mathrm{s}$. 
The analyses with combined CF and IL loading did not give good response estimates for the remaining tests in the short span test series 42 and 75 . These experiments had CF and IL mode 1 response. The previous version of VIVANA is incapable of predicting this combination of modes and gave $\mathrm{CF}$ and IL response frequencies which were too high. Based on the aforementioned limitations, the present coefficient based approach with no interaction between the $\mathrm{CF}$ and IL response calculations looks promising.

It was recommended that further work be carried out to have an understanding and enable prediction of the CF and IL frequency and mode interaction. The CF and IL mode 1 cases are of particular interest.

Recent works by Yin et al. (Yin, Passano, \& Larsen, 2018), have shown that in the recently updated version of VIVANA, the prediction of IL responses for combined IL and CF VIV responses has been improved.

\subsection{SHEAR7 ${ }^{\mathrm{TM}}$}

SHEAR7 is a mode superposition program developed in MIT (Shear7, 2018), which evaluates which modes are likely to be excited by vortex shedding and estimates the steady state, crossflow, VIV response in uniform or sheared flows. Shear7 is one of the prominent modelling tools for predicting VIV. This program is ideal in predicting offshore pipelines under the action of spatially varying currents found in ocean environments. Pure in-line VIV analysis for pipelines have recently been incorporated in the SHEAR7 v4.7. Structural responses, mean drag force amplification factors and fatigue damage can be estimated using SHEAR7 (Shear7, 2018).

\subsection{FatFree $^{\mathrm{TM}}$}

Free span assessment is extremely complex as it requires detailed knowledge in several disciplines (structural response including geotechnical aspects, environmental conditions, long-term statistics, etc.). The DNV RP F105 is still complex and difficult to use. Therefore a 
calculation tool is necessary to make it easier to apply the recommended practice, enable a cost efficient span assessment, etc. FatFree is a professional engineering analysis software based on the state of the art principles of the DNV RP F105.

The DNV RP F105 applies the Response Models approach to predict the vibration amplitudes due to vortex shedding. These response models are empirical relations between the reduced velocity defined in terms of the still-water natural frequency and the non-dimensional response amplitude. Hence the stress response is derived from an assumed vibration modes with an empirical amplitude response (Det Norske Veritas, 2002).

According to Xing (Xing, 2011), SIMLA was used to perform eigen-mode analysis for 3 inline and 3 cross-flow models. Subsequently, the eigen modes obtained was used with the FatFree to calculate fatigue damage along the pipeline due to VIV.

This was achieved by establishing a free span model using SIMLA for given pipeline incorporating terrain data, pipeline diameter and thickness, coating and flow characteristics and environmental conditions, and then perform static analysis including all phases of pipeline behaviour, i.e. installation, water filling, hydrostatic testing, etc. and then again analyse the eigen mode for 3 in-line and 3 cross-flow models. For a given sea state, the new FATFREE was then utilised in conjunction with the eigen modes obtained from SIMLA to compute the fatigue damage.

He described SIMLA as a computer program which allows for both nonlinear static and dynamic analysis such that in both cases time domain is employed in describing the load histories and analysis sequence. Several features have been integrated such as new element types and non-linear time domain dynamics, but SIMLA does not have the capability of handling VIV. 
Also, FATFREE was described as a Microsoft excel VBA spreadsheet established by DNV for design and (re-)assessment of submarine pipeline spans. FATFREE calculates the fatigue life capacity due to combined direct wave action and inline VIV and cross flow VIV.

In the same report, simplified ULS design checks in terms of peak stress and equivalent stress due to combined static and dynamic actions were provided.

The result analysis as was presented shows that the eigen frequency corresponding to the first eigen mode at cross flow $=0.54$, for second eigen mode at cross flow $=1.39$, etc. It was stated that the data was computed based on the pipeline configuration for operation load condition. It was claimed that for the natural frequencies, no significant VIV damage supposed to occur in the bottom current velocity range considered. Industry leaders in free span analysis include INTECSEA and JP Kenny.

\subsection{INTECSEA's assessment tools}

Pereira et al, (Pereira, Franco, Tardelli, Bomfimsilva, \& Eigbe, 2008), established a methodology and suite of FE based tools for multi-mode/ multi-span VIV fatigue assessment in order to overcome the limitations inherent in the approximate response models (i.e. single spans with levelled shoulders, short length - span lengths $<140 D$, etc.) particularly for 'long' and multi spanning pipelines based on the DNV RP F105 recommendations on the calculation of natural frequencies, mode shapes and stresses associated with these mode shapes for the assessment of VIV fatigue via eigen value analysis.

The conventional methods of estimating MAFSL to avoid VIV normally involves a large number of free span correction, which may be too conservative for the project. It was claimed that this approach was used in recent projects to achieve better estimate of the requirements for free span correction thereby saving cost and has the potential for assessment of pipelines just after installation as well as existing pipelines during operation where new spans created due to soil movements are usually observed via inspection over the design life of the pipeline. 
The authors examined the in-place FE methodology, conducted a validation process and used the tools established to accelerate the fatigue assessment procedure which is claimed crucial to the determination of free span correction requirements in the field particularly whilst analysing the post lay survey process in real-time. The steps involved in the methodology are bottom roughness analysis, modal analysis and fatigue analysis:

\subsubsection{Bottom roughness analysis}

This is performed to determine the initial static equilibrium configuration and expected loading along the pipeline in the as-laid, temporary and operational conditions.

This involved the use of FE program referred to as SPAN-CALC ${ }^{\mathrm{TM}}$ (an elastic pipe element which was developed in ANSYS employed to model the pipeline. The spring combination element was employed in modelling vertical seabed stiffness and axial and lateral friction represented by nonlinear springs for the pipesoil interaction, etc..

It also involved the use of a SPAN-CALC ${ }^{\mathrm{TM}}$ post-processor consisting of an excel spread sheet incorporating visual basic application for processing the results from the bottom roughness analysis for identification of critical spans and ascertain their overall characteristics, etc.

\subsubsection{Modal analysis}

Here, MODE-CALC ${ }^{\mathrm{TM}}$ was used for the eigen-value analysis developed in ANSYS in order to identify the contributing vibration modes which may be activated during the pipeline's life on the basis of expected current velocities. This analysis involved taking into account the fact that adjacent interacting spans from near vicinities can considerably affect the span vibration; selection of mode shapes based on the assumption of maximum curvatures arising from unit amplitude displacements.

A MODE-CALC post-processor developed in excel with associated visual basic application was adopted to retrieve and select mode shapes and the natural frequencies derived from the modal analysis. 


\subsubsection{Fatigue assessment}

This was performed with the use of FATCALC ${ }^{\mathrm{TM}}$ developed using the MathCAD software. It is also supported by excel and visual basic application to extract results from the modal analysis and calculate the damage to the pipeline in the temporary and operational phases. For each critical span, the procedures adopted include: The determination of the maximum nondimensional response amplitudes according to the DNV RP F105 specification.

Calculating the actual (de-normalized) in-line and cross-flow displacement amplitudes along the span, calculation of the pipeline curvatures $k(x)=1 / \rho(x)$ based on the actual displacement amplitudes using a finite difference algorithm, etc.

Comparisons were made between the result from the proposed methodology and associated tool with those obtained from FATFREE (version 10.0). The validation exercise included two tasks: first to verify the natural frequencies and peak curvature from the modal analysis program and the second was to verify the calculated fatigue life of free spans obtained from the fatigue calculation tool.

It was noted that the survey data for soil and oceanographic information has to be reliable for an effective application of this methodology. Also, the definition of the requirement for free span correction can only be determined as soon as post lay survey is available, since information applied during the design stage extracted from geophysical survey can differ substantially from the as-laid conditions, as the as-laid survey will capture the actual pipeline configuration depending on the field specific seabed conditions.

Finally, a conclusion was made that the different tools including the SPAN-CALC, MODE CALC and the FATCALC as described have been seamlessly integrated to facilitate an automated and quick implementation of the fatigue damage assessment procedure especially in cases of pipelines having a huge number of free spans requiring detailed assessment. Applying this procedure and corresponding tools in isolated fashion could become tedious and 
unmanageable, specifically in a live project setting. In addition, the seamless integration of these tools is also vital for field situations requiring quick response in real-time decision making to determine the requirements for correction of free span identified during post-lay surveys.

\subsection{JP Kenny's Assessment tools}

According to Jukes et al (P. Jukes, Eltaher, Wang, \& Duron, 2008; Paul Jukes, Wang, \& Duron, 2008), the Simulator (runs on ABAQUS engine) is an advanced FEA tool that allows accurate prediction of pipeline response. The models include elasto plastic materials, 3D route geometry, peak and residual modelling of axial and lateral soil pipe forces. It was reported that PIP and single pipe models were developed.

The 'simulator' analysis was described as static large deflection analyses which include all relevant non-linearities such as large deflections and large rotations, elasto-plastic pipe materials interpolated over relevant temperature ranges, and non-linear pipe soil interactions. The application of the Simulator during the design stage permits limit state based designs as well as the following: change and optimize the design, undertake a range of sensitivities, optimize the design, etc. The design can be iterated and design optimisation can be achieved via the adoption of limit states such that significant financial savings may be achieved.

It was reported that the simulator was designed to analyse the initial, prior to instability moment and post lateral buckling and expansion behaviour of straight, single pipe in pipe system flowline lying on flat seabed. The model was claimed to be applicable for shallow or deepwater condition and/or HTHP PIP system. The module was reported to be able to perform parametric studies if required, by simply changing the input parameters of the input script code. After, the completion of single analysis, the following results are presented: submerged weight, DNV load controlled utilization, etc. 
The FE elements used are the hybrid formulation pipe elements within ABAQUS. These elements are selected, as they are particularly well suited to modelling long, slender pipelines with better convergence behaviour than the standard pipe elements. Other detailed descriptions can be found in Jukes et al. (Paul Jukes et al., 2008).

Sun et al. (Sun, Jukes, \& Wang, 2011) discussed the use of 2-D FEA for the identification of free spans and described the 3-D pipeline FEA as being a more reliable tool for span analysis for HP/ HT pipelines since global buckling will have a significant impact on free spanning behaviour. 3-D FEA captures the pipeline uplift at the crest, the pipeline downward at the span, and the change of global buckling plane from vertical to horizontal. These programs are nonlinear and based on the ABAQUS. Sun et al. (Sun, Jukes, \& Duan, 2009), used the Simulator ${ }^{\mathrm{TM}}$, a FEA tool based on ABAQUS, to ascertain the relationship between the pipeline free span dynamics and the thermal expansion/ global buckling inherent under the HP/ HT conditions. A single pipeline and Pipe-in-Pipe flowline both were studied. The analysis laid emphasis on the conditions which could enhance the pipeline "Feed-in" into the span and the variations of effective axial load at the free span positions. As a unique lateral buckling and free span interactive situation, the dynamics of sleeper span was also examined.

The "Simulator", a JP Kenny in-house finite element engine based on ABAQUS, is often used to develop pipeline analysis models including the free span dynamic analysis. A FEA with ability of simulating Pipe-in-Pipe instead of the conventional equivalent single pipe was applied. The 2-node pipe element, PIPE31H, was adopted for the formation of both inner and outer pipe which is a hybrid pipe element formulation within ABAQUS. This element type is ideal for modelling long, slender pipelines with better convergence behaviour than the standard pipe elements. An elastic connector element was applied in the simulation of the bonding of the end bulkheads which are generally implemented in the subsea structures and riser-flowline transition point which convert the PIP into a single pipe section. The inner pipe centralizers' 
interactions (which are clamped on the inner pipe at designated spacing) and the outer pipe were modelled via utilizing a tube-to-tube element (ITT) which permitted the axial and lateral movements since they are constrained by the clearance and the friction. The ITT element accurately simulates the contact reaction and load transfer between the inner pipe (via the centralizer) and the outer pipe during the installation and operation.

The following were the conclusions drawn based on the study:

(a) As the thermal expansion proceeds, free span dynamics varied significantly. This is apparent since the span natural frequencies are a function of effective (compressive) axial force which interprets the effects of operation temperature and pressure. The study revealed that thermal expansion due to high temperature can change a single span into interactive spans and during the process a sudden change of the frequencies of in-line modes can occur. The fundamental natural frequency of the in-line oscillation mode can be smaller in value even if the span length is shortened.

(b) Thermal expansion can form a rigid central boundary for a low gap free span and result in a much higher fundamental natural frequency.

(c) The fundamental dynamics of 1 st cross-flow mode is more sensitive to the thermal expansion. The mode shape can change from single span behaviour to interactive span behaviour in low operation temperature and the frequency may rather increase.

(d) The sleeper reduces the lateral resistance and encourages the lateral buckle. As a result, the 3D mode shapes can be the fundamentals for the sleeper formed interactive span.

Using an equivalent single pipe to model the sleeper span of a PIP can end up an unsafe design analysis although it can provide the correct in-line and cross-flow dynamics. In some cases, it fails to catch up the lower natural frequencies that may be in 3-D mode shapes.

Apart from the fact that their study concentrated on the high pressure and high temperature operation, it also demonstrated that FEA based analyses are indispensable as recommended by 
the design code for analysing free span dynamics. Also, it was claimed the study further demonstrates the uncertainties of free span dynamics as it interacts with the flowline operation particularly under the high temperature and pressure.

\subsection{MCS Kenny's Assessment tools}

Wang et al. (Wang, Xu, \& Jukes, 2010) presented a methodology for span analysis in view of the onerous nature of free spanning pipeline analysis which involved a simple screening, with intermediate screening followed by a detailed FEA. This approach was claimed to be efficient since it not only make sure all span issues are captured but also minimizes superfluous calculations. Also the approach was acclaimed such that issues like overlooking critical pipeline spans are prevented. The applications include existing versus new pipelines, selection of pipeline route versus as-installed analysis, pipelines with and without thermal buckles, and an analysis with complete versus incomplete seabed survey data. The method has been claimed to serve as a guideline for a cost effective span analysis.

Based on the MCS Kenny In-house experience, the tools used in the screening analysis are often established in spreadsheets incorporating DNV equations which must be able to conduct iterative calculations. In the detailed analysis, static FEA models and spreadsheets can be applied for the ULS check and dynamic FEA models and DNV FATFree software used for fatigue calculations.

The spreadsheet for the detailed analysis calculates the VIV induced moment from the unit stress obtained from the FEA model, obtains the direct wave-induced moment from the FEA model. These are factors distinguishing the detailed from the spreadsheets for screening analyses.

FEA modelling is applied in the detailed analysis. This is divided into the static and the dynamic phases. The static phase determines the sag deflection under the operating conditions as well as the pipe-soil interaction modelling adopting the node-to-surface contact using 
ABAQUS. The contact model includes two contact surfaces: the pipe surface and the seabed surface, the pipe and soil interaction in the vertical direction is modelled using the stiffness defined as linear pressure over-closure relationship with an upper limit based on the soil bearing capacity among other features.

According to Wang et al. (Wang et al., 2010), the recent version of the DNV FATFREE includes the effects from direct waveload effect and interacting spans. It was also noted that while performing fatigue calculations it is apposite to use mode shapes in FatFree instead of unit stress as the worst position is normally not clear for interacting span.

Wang et al. (Wang et al., 2010), also described the span analysis for an existing as well as a new pipeline where screening and detailed analysis (including ULS check with DNV and ASME codes, seabed support, global buckling etc.) were discussed. The author made the assertion that the methodology could be applied as a starting point for projects with complicated spans.

Wang et al. (Wang et al., 2011), described the latest developments in the use of finite element analysis to examine associated mitigation solutions given the dictating practical limitations and cost factors. This was carried out in order to optimize mitigation methods such that unnecessary works or concerns in future are avoided. The ULS and fatigue life criteria were adopted as guidelines.

In their work, the ULS mitigation was first carried out when both ULS and fatigue life improvement are planned, as the ULS mitigation methods commonly have positive impact on the span fatigue life. The mitigation measures considered for each span included mechanical supports, grout bags, sand bags, and strake for VIV and concrete mats. The above mentioned were evaluated in terms of their advantages and limitations regarding each spans involved. The selected mitigation method is then analysed for its effectiveness by simulating it through FEA 
using ABAQUS software. The DNV FATFREE is employed for the fatigue life calculation and the ULS of the pipelines is then found using an MCS Kenny's in-house spread sheet.

Several conclusions were made some of which include:

(a) Details on span analysis and fatigue analysis were presented with emphasis on the span mitigation analysis.

(b) Several span mitigation case studies were used to demonstrate selected mitigation approaches with assistance of advanced FEA techniques.

(c) The results of the case studies revealed that span mitigation using grout bags and mechanical supports can successfully improve the ULS value and fatigue life.

(d) The use of advanced FE modeling for span mitigation at early design stage allows identification of unique challenges associated with each span and selection of the optimal mitigation methods.

(e) Various limitations associated with any selected mitigation approach have to be accounted for in the FEA to find a practical mitigation solution.

(f) The installation sequence and the tolerances are to be considered in the FE simulations as their impact, especially on ultimate limit state value, can be significant.

For the FEA modelling, ABAQUS could be utilised to simulate the span structure both in static and dynamic phases. This includes the PIPE31H element and performs the same function as described previously.

Typical example case study result for span mitigation by grout bags is as follows: for given span characteristics, the gap at the mid-span was $<1 m$ and the recommended mitigation solution was thus the application of grout bags as support. It was claimed that the fatigue life after mitigation was improved to an acceptable result and the ULS check was unchanged as the pipe curvature remained about the same. 
The span mitigation analysis by supports only yielded a final mitigation strategy involving two steps such that fatigue lift is improved to over 50 years. The span mitigation by supports with TLS assisted was also covered.

Wang et al. (Wang, Jukes, Wang, \& Duan, 2008), described a FEA program that was established to simulate a flowline span response under complex loading and boundary conditions. Both direct wave loading and VIV were captured in the analysis and the results were sequentially used for the fatigue life calculation and ULS check. It was noted that FEA is crucial for a successful span assessment as it was usually applied in computing parameters required for fatigue life and ULS calculations such as natural frequencies, unit stresses and mode shapes precisely. In view of the simplicity and accuracy offered by 2-node pipe elements, it was used to model the pipeline.

According to the same author, FE modelling of span analysis is divided into static and dynamic phases. In the static phase, the determination of the sag deflection under operating conditions while the dynamic phase deals with resolving the natural frequencies and corresponding mode shapes and the use of springs to model the pipe-soil interaction. The dynamic phase can be said to be a linearized procedure which point towards linear effects, and any nonlinearity such as friction, etc. are not considered. Thus, spring elements alone are applied to model the dynamic soil stiffness.

The methodology includes several procedures such as definition of the model length, fluid mass consideration, concrete modelling, etc. among other details. The following where the conclusions made: (a) A practical methodology for analysing free span pipelines was presented. (b) With good apprehension of the DNV RP F105, advanced numerical FE tools simulate pipeline span dynamic and static phases adequately. Factors such as element size, model length, 
fluid mass consideration, concrete induced SCF (Stress Concentration Factor) at field joints, etc. are determined with special care in FE modelling.

It was noted that in the ULS check, the bending moment is very sensitive to lateral friction particularly for interacting spans with very narrow shoulder; the assessment of the slugging condition is required - slugging could increase or decrease the ULS results; the worst condition needs to be identified with variation of concrete degradation and soil stiffness; and whilst considering the wave/ current directionality, metocean magnitude tolerances and direct wave loading effects special care has to be exercised. The author also asserted that this methodology can be adopted as a starting point for projects with complicated spans.

\subsection{Assessment tools for multi-spanning subsea pipelines}

Rezazadeh et al. (Rezazadeh et al., 2010), proposed a VIV fatigue analysis approach for multi spanning pipelines based on VIV analysis in view of the unclear, undetailed DNV RP F105 (Det Norske Veritas, 2006) dynamic analysis methodology suggestions. The author noted that the pipe soil interaction - continuous two span gap controls multi spanning pipeline fatigue damage. The Abaqus FE model was developed in order to first ascertain the distribution of stress as well as the natural frequency of each vibration mode for spanned pipeline on several bedforms characterised by three multispans. Subsequently, a VIV fatigue analysis is conducted for the spanning pipeline to estimate the fatigue life capacity due to the stress and eigen frequencies obtained from Abaqus analysis for the in-line and cross-flow directions according to DNV RP F105 (by application of a mathCAD worksheet the stresses and frequencies being inputs). Based on sensitivity analyses results, the effects of significant parameters on VIV fatigue were demonstrated:

It was claimed that only the initial modes are either dominant or participating, the modes with the highest frequency tending to result in very slight pipeline damage in the multi span pipeline fatigue damage evaluation case; normally low current flow velocities are neglected for the 
muti-span pipeline fatigue life assessment because of insignificant contribution to pipeline multi span fatigue damage.

According to the same author, the pipeline was modelled as a 3D beam; the supports were modelled with rigid surfaces by ignoring the pipe embedment for conservatism; applying axial fixity at both ends after pipeline installation taking into consideration the bottom tension force. Application of load steps was in the following sequence: (1) gravity load (2) internal pressure (3) external pressure (4) temperature to match the effective axial force.

Once the model has been established, the apposite loading conditions, natural frequencies in the inline and cross flow directions and corresponding mode shapes can be ascertained from the frequency extraction test.

\section{Comparative studies}

\subsection{Comparative Studies among Models for Predicting VIV}

The Table 2 shows the capabilities and limitations of the different models applied for predicting VIV for pipelines in operation in deep water. Most semi empirical models have until recently been limited to Cross Flow (CF) VIV due to lack of hydrodynamic coefficients for IL response [137].

According to Durowoju (Durowoju, 2012), in a study which showed comparison between the semi-empirical, CFD and Orcina models, there were variations in the results displayed by the models. For the empirical models only VIVANA and ABAVIV predicted in-line displacement and the cross-flow displacement predicted by them were closer to those predicted by the CFD based codes.

The maximum in-line displacement was under-predicted by all the models. It was also noticed that the CFD based prediction of cross-flow displacement and curvatures are considerably smaller than measurement. The numerical models that use the frequency domain identified one or more frequency at which cross-flow response occurred. With the exception of ABAVIV, 
most frequency from the empirical based models, VIVA, VIVANA, VICOMO and shear7 follow the same behaviour and all were close to the measured frequency.

Also from the same study, the prediction of cross-flow displacement from the semi-empirically based models were between $85 \%$ and $100 \%$ compared to the actual measurement except for shear7 that displayed more than $100 \%$. The Orcina wake oscillator displayed between $85 \%$ and $140 \%$ of the corresponding measurement. The CFD codes on the other hand were characterised by smaller cross-flow displacement in the range of $65 \%$ to $90 \%$.

Only the Orcina vortex tracking model over-estimated dynamic in-line displacement and the in-line and cross-flow curvatures were by $100 \%$ or more. The empirical models with the exception of the orcina vortex tracking models which over-estimated the response were more successful in predicting the cross-flow displacement which is the major contributor of fatigue for deepwater risers. A breakdown of the results is shown in the Table 1 below.

Table 1: Comparing numerical models (Durowoju, (Durowoju, 2012))

\begin{tabular}{|c|c|c|c|c|c|c|}
\hline \multicolumn{2}{|c|}{ Numerical models } & \multirow{2}{*}{$\begin{array}{l}\text { In-line } \\
\text { displ. } \\
\text { No }\end{array}$} & \multirow{2}{*}{$\begin{array}{l}\text { Cross - } \\
\text { flow displ. } \\
\text { Yes }\end{array}$} & \multirow{2}{*}{$\begin{array}{l}\text { Peak cross } \\
\text { flow disp. } \\
\text { B }\end{array}$} & \multirow{2}{*}{$\begin{array}{l}\text { Maximum } \\
\text { in-line } \\
\text { displ. } \\
\text { A }\end{array}$} & \multirow{2}{*}{$\begin{array}{l}\text { Frequency } \\
\text { and mode } \\
\text { numbers }\end{array}$} \\
\hline Semi- & Shear7 & & & & & \\
\hline empirical & VIVA & No & Yes & $\mathrm{A}$ & $\mathrm{A}$ & $\mathrm{B}$ \\
\hline & VIVANA & Yes & Yes & B & $\mathrm{A}$ & $\mathrm{B}$ \\
\hline & VICOMO & No & Yes & A & A & $\mathrm{B}$ \\
\hline & ABAVIV & Yes & Yes & A & A & A \\
\hline \multirow[t]{4}{*}{ CFD } & $\begin{array}{l}\text { Norsk } \\
\text { hydro }\end{array}$ & Yes & Yes & A & A & A \\
\hline & USP & Yes & Yes & A & A & A \\
\hline & Deepflow & Yes & Yes & A & A & A \\
\hline & VIVIC & Yes & Yes & $\mathrm{A}$ & A & A \\
\hline \multirow[t]{2}{*}{ Orcina } & $\begin{array}{l}\text { Vortex } \\
\text { tracking }\end{array}$ & Yes & Yes & $\mathrm{C}$ & $\mathrm{C}$ & A \\
\hline & $\begin{array}{l}\text { Wake } \\
\text { oscillator }\end{array}$ & No & Yes & $\mathrm{B}$ & $\mathrm{A}$ & $\mathrm{B}$ \\
\hline
\end{tabular}

A - Prediction smaller than measurement

B - Prediction in agreement with measurement 
$\mathrm{C}$ - Over-estimated compared to measurement

The Author claimed that the semi-empirical models are widely used in the industry and that though the CFD use a more advanced approach, its implementation in the industry is still growing. The in-line displacement becomes a major concern especially when it has to do with free span pipelines. From the experiment most of the CFD models predicted in-line displacement but were in poor agreement with the measurement.

More recently, Dos Santos et al. (Dos Santos, Morooka, Caire, Franciss, \& Matt, 2014) conducted a comparative study on experimental and numerical simulation results for displacements of a pipe with free span. The study focused on cross-flow vibrations, and simulations were carried out through different computer programs and, respective VIV models. Numerical simulations on different current velocity conditions and calculations by varying model parameters were conducted. The study results revealed that, on the average, the overall condition computed by all programs, calculated cross-flow displacements were between $69 \%$ and $200 \%$ of the corresponding experiment data.

In the time domain, the OrcaFlex by Orcina had shown very conservative results, overestimating the dynamic cross-flow amplitude of vibrations by $200 \%$. The Author suggested that, perhaps, additional investigation is desired regarding appropriate adjustment of stiffness and damping reduced coefficients, in order to improve results in comparison to experimental ones. The ANAPIPE-VIV presented good result when the Strouhal number was set equal to the response frequency from the experiment, on average by $97 \%$ of the corresponding measured data in the experiment. However, both ANAPIPE- VIV and RiserProd were not satisfactory for the lock-in range. In order to improve calculations, it was concluded that reliable empirical coefficients are needed. 
VIVANA in the frequency domain, by using default parameters, has shown good agreement with the experiment with average of $111 \%$ of the corresponding experiment data set.

Shear7 by using constant Strouhal number and for values equal to 0.18 has shown result on the average, by $73 \%$ of the corresponding experimental data set and, when the Strouhal number was set to be the same as experiment, it was on average by $134 \%$.

In conclusion, all the programs do not represented with good agreement the lock-in region located for $V_{R}$ between 6 and 7. Simulations underestimated the cross-flow amplitude of vibration, in general, and further investigation is still needed for this region of $V_{R}$.

Consequently, most of the programs use empirical and semi empirical approaches to estimate VIV forces based on measured coefficients in laboratory or field tests, such as the lift coefficient and Strouhal number. However, further laboratory experiment as well as field test data measurements are still needed to clarify and to support better estimation of VIV hydrodynamic forces.

Table 2: Capabilities and Limitations of most common VIV prediction models

\begin{tabular}{|c|c|c|}
\hline $\begin{array}{l}\text { Numerical } \\
\text { Models }\end{array}$ & Capabilities & Limitations \\
\hline DNV-RP-F105 & $\begin{array}{l}\text { Predicts cross flow and in-line forces } \\
\text { (Det Norske Veritas, 2006). }\end{array}$ & $\begin{array}{l}\text { Its IL VIV curve in the } \\
\text { Frequency vs Amplitude ratio } \\
\text { graph is an envelope curve } \\
\text { (Aronsen, Larsen, \& Mork, } \\
\text { 2005). The results from the } \\
\text { studies carried out by Koushan } \\
\text { (Koushan, 2009) on the time } \\
\text { domain analysis with compare to } \\
\text { the frequency domain show how } \\
\text { important is to consider the } \\
\text { difference between the behavior } \\
\text { of a free span pipeline modeling } \\
\text { in linear or non-linear analysis. } \\
\text { However, this fact has not been } \\
\text { accounted by the guidelines yet } \\
\text { such as the DNV RP F105. In a } \\
\text { comparative study, It was } \\
\text { observed that results from tests }\end{array}$ \\
\hline
\end{tabular}


VIVANA

Shear 7

Computational

Fluid Dynamics

(CFD)

Wave Oscillator

Model
Predicts inline and cross flow forces (Passano et al., 2010). The model has been updated which now produces improved in-line VIV prediction for combined in-line and cross flow VIV responses (Yin et al., 2018).

Predicts cross flow and pure in-line forces. Pure in-line VIV analysis for pipelines have recently been incorporated in the SHEAR7 v4.7. Well known to predict CF VIV accurately as evidenced in its widespread use for VIV prediction in risers (Shear7, 2018)

Predicts cross flow and In-line displacements. The CFD use a more advanced approach. CFD simulation of turbulent fluid flow around one or several pipes can in principle be applied for VIV assessment to overcome the inherent limitations of the state-of-practice engineering approach

Xu et al. (W. H. Xu, Gao, \& Du, 2012) developed a wake oscillator model capable of analysing the pure IL VIV of slender marine structures. It was claimed that the result show the model predicts can reproduce some of the pure IL VIV as obtained in experiments. generally show lower response in comparison with the DNV response prediction (Koushan, 2009).

There is still a need to enrich hydrodynamic coefficient database, Optimize the updated coefficient database and calibrate/ optimize the updated coefficient database against other flexible model test.

Information gathered shows that it has limited use in analysis of pipeline free spans (Durowoju, 2012)

The application for VIV assessment is at present severely limited by the computational effort required (Det Norske Veritas, 2006). Its implementation in the industry is still growing.

This model is limited to structures with only pure IL VIV phenomenon

\subsection{Comparative study on the use of FEA in span analysis with field data}

It is important to reliably predict the pipeline profiles in order to precisely assess the pipeline free span response and to provide guidance to determine a proper free span mitigation strategy. The FEA method can be utilized to realistically simulate the pipeline on-bottom roughness behaviour affected by pipeline properties, pipe-soil interaction including penetration and soil 
friction resistance, the internal and external pressure, product content, temperature profile and bottom tension from pipe-lay. An FEA model which is accurately built can provide a reliable prediction for the pipeline profile, free spanning length and gap under all conditions, which are crucial for stress and fatigue assessment as well as for the design of free span mitigation (S. Huang, Sun, Abdalla, \& Group, 2017).

In (S. Huang et al., 2017) the result of a comparative study between FEA and as-laid pipeline profiles shows that there was correlation for the empty, flooded, post-hydrotest and operating conditions as the pipeline profile, free spanning length and gap predictions were accurate.

\section{Case Study}

Figure 2 presents a flow chart describing the free span assessment procedure. Figure 3 depicts the section of the pipeline assembly showing the different parts. When subsea pipelines are designed, it is important to determine the maximum allowable free span length (MAFSL) that will be acceptable during its operation under different environmental conditions to ensure that spans generated do not exceed this allowable length. The calculation of the MAFSL is done to identify any possibility of the occurrence of a free span during operation and eliminate the occurrence by ensuring proper design and construction (Guo et al., 2005; Shittu et al., 2012). 


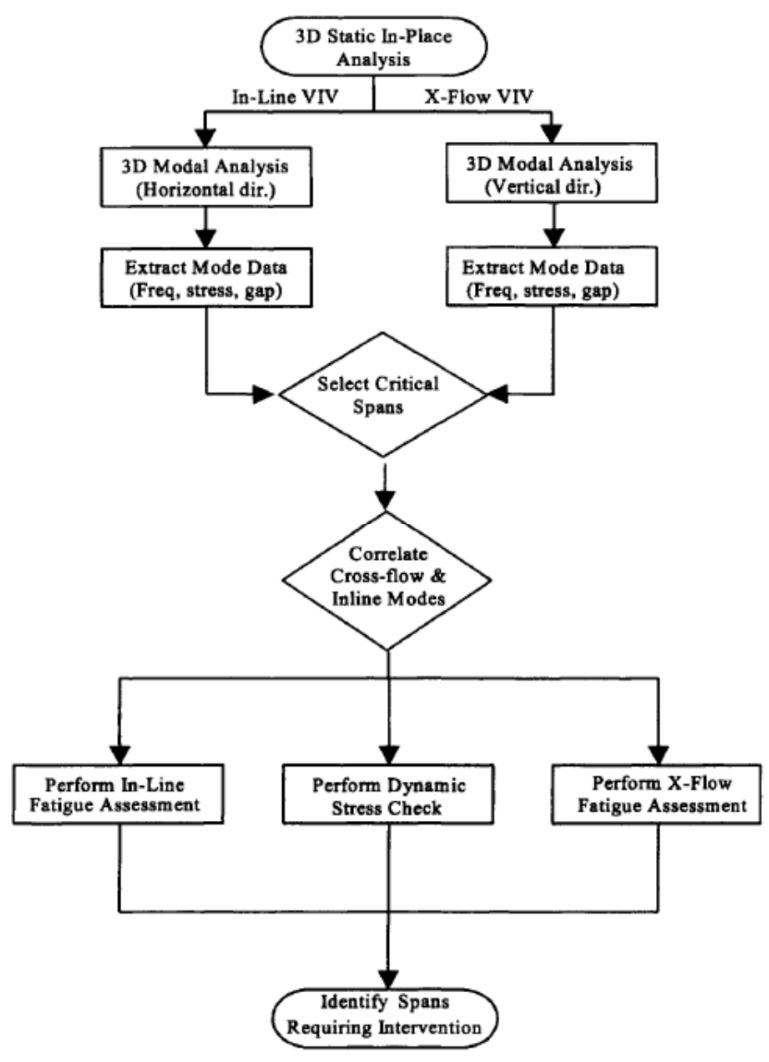

Figure 2: Flow chart describing the free span assessment procedure. Souce: Bai and Bai (Bai \& Bai, 2010).

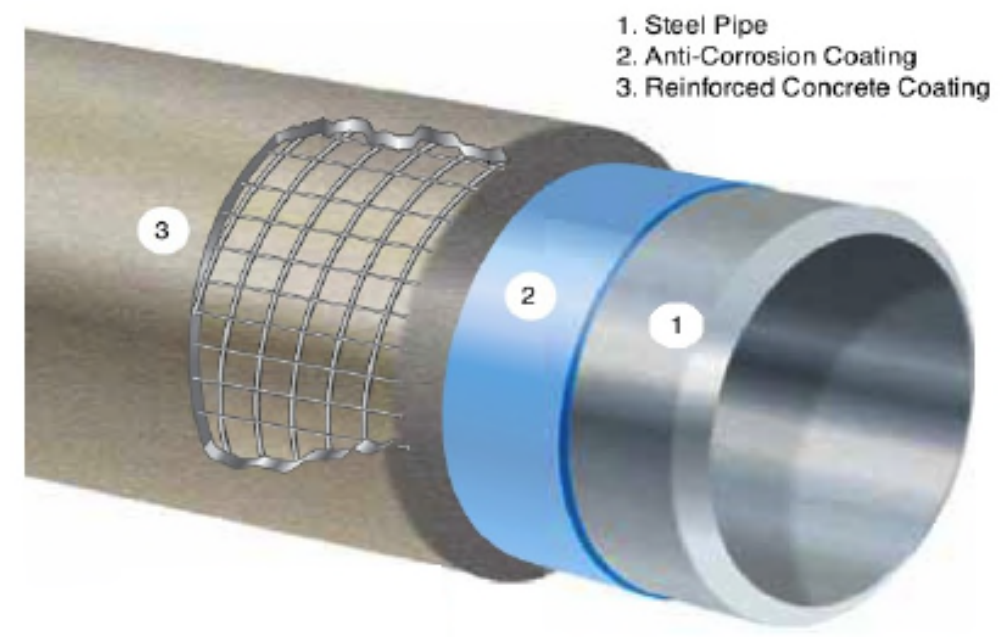

Figure 3: Section of Pipeline assembly showing the different parts. Source: Bredero Shaw (Brederow Shaw, 2012) 
The local pressure distribution in the vicinity of a pipeline is altered whenever a vortex is shed, and a time-varying force is experienced on the pipeline at the vortex shedding frequency. Pipelines can fatigue from oscillation which can lead to failure. These oscillations can be generated from resonant conditions which can make the pipeline oscillate continuously at a frequency. The pipeline can oscillate either in-line with the flow direction or transverse (crossflow) to the flow direction by the action of vortex shedding, depending on span length and current velocity.

In-line Oscillations: In-line oscillations are generated at flow velocities lower than the critical velocities for cross-flow motion and they have amplitude of only $10 \%$ of amplitudes for crossflow motion. It is suggested that the stability parameter $K_{s}$ be greater than 1.8 , to prevent inline response at either mode of vortex shedding generation/action (Guo et al., 2005). According to DNV (Det Norske Veritas, 2006), resonant in-line vortex shedding induced oscillation may occur if the reduced velocity is in the range $1.0<V r<2.2$, and stability parameter $K s<$ 1.8, for this case, the vortex shedding will be symmetrical. For the $V r>2.2$, the vortex shedding will be alternate or asymetrical.

Cross-flow Oscillation: The potential danger for disturbances or excitations in the cross-flow direction is more severe than for those associated with the in-line direction because the response amplitude for crossflow is great than those associated with in-line motion. The limiting value for cross-flow oscillation based on DNV is $V r>3.5$ and $K s<16$ (Det Norske Veritas, 2006)

Design Steps: The design steps for determining the allowable pipeline free span length due to dynamic forces was proposed by Guo, et al. (Guo et al., 2005) as depicted in Figure 4 


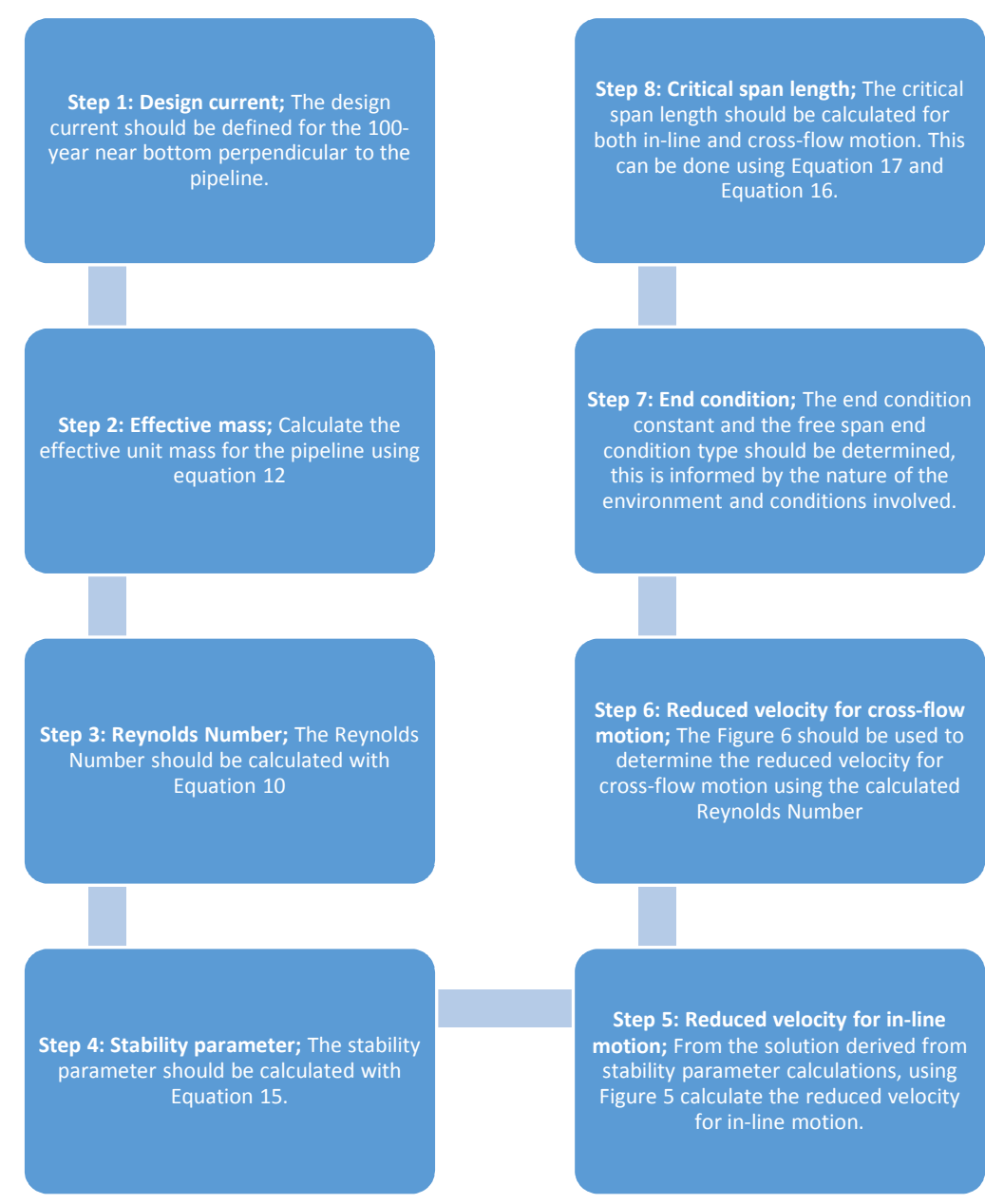

Step 9: Allowable span length; The critical span length determined for inline motion is usually selected for several project design as the allowable span length. The cross-flow motion however may be used when permitted by some economic factors.

Step 10: Fatigue life; The fatigue life of the free span should be calculated and analysed if the in-line motion is permissible.

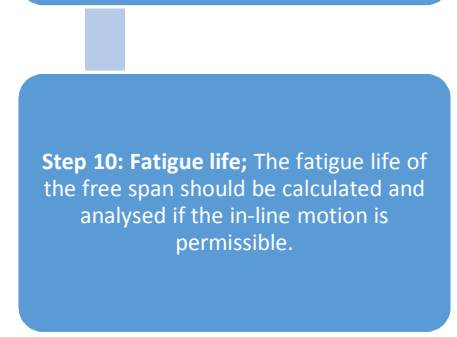

Figure 4: Design steps for determining MAFSL

\section{Design Considerations}

According to Guo, et al. (Guo et al., 2005) the design considerations to be considered is outlined as follows:

\section{Dynamic Stresses}

Substantial dynamic stresses on a pipe can result from the presence of bottom current when the pipeline oscillates as a result of vortex shedding. This has an adverse effect on the pipeline weld because the oscillation can cause weld area fatigue and the pipeline life can be reduced. 
Functional loads that may produce Minor dynamic amplification of this action is considered in the static analysis. Certain conditions such as correct sequence of loading and soil-pipe interaction effects are included in the analysis.

The pipe span will vibrate by resonance effect when there is a synchronization between the vortex shedding frequency and one of the natural frequencies of the pipe span. The frequency of vortex shedding also known as Strouhal frequency is a function of the diameter of the pipe, current velocity and strouhal number.

Free spanning pipelines should have a level of safety adequate enough against fatigue, fracture, local buckling and ovality (Zaki, 2006). The in-line and cross-flow motions of a free span length can be calculated using the steps given in (Bai, 2001, 2003, Bai \& Bai, 2005, 2010; Chakrabarti, 2005; Guo et al., 2005; Jain, 2012) as follows.

Vortex shedding frequency: This is also known as strouhal frequency and it's the frequency at which vortices pairs are shed from a pipeline. It can be calculated from the equation (9):

$$
f_{s}=\frac{S V_{c}}{D_{o}}
$$

The strouhal number is a function of Reynolds number and it is the dimensionless frequency of the vortex shedding. Reynolds number is a parameter that represents the ratio of inertial force to viscous force, and is expressed as:

$$
R_{e}=\frac{V_{c} D}{v_{k}}
$$

For sea water, $v=1.307 \times 10^{-6} \mathrm{~m}^{2} / \mathrm{s}$

Natural frequency of a pipeline: The pipeline spans natural frequency depends on some factors like the effective mass of the pipe, end condition of the pipe span, length of the span and the pipe stiffness. Equation (11) gives the natural frequency for vibration of the pipe span: 


$$
f_{n}=\frac{C_{e}}{2 \pi} \sqrt{\frac{E I}{M_{e} L_{s}^{4}}}
$$

The effective mass of the pipe $M_{e}$ is the sum of total unit mass of the pipe content, the unit mass of the pipe, unit mass of corrosion and concrete coating, and the unit mass of the displaced water which is called the added mass. This is given as:

$$
M_{e}=M_{c}+M_{p}+M_{c o r r}+M_{c o n c}+M_{a}
$$

The added mass is the mass of water that has been displaced by the pipeline. The value can be determined from the formular:

$$
M_{a}=\frac{\pi D_{o}^{2} \rho_{w}}{4}
$$

For seawater, $\rho_{w}=1025 \mathrm{~kg} / \mathrm{m}^{3}$ Or 2 slugs $/ \mathrm{ft}^{3}$

The end condition constant $C_{e}$ Is determined by the type of model used to evaluate the support conditions of the pipeline span. Based on these end conditions, the following values are used:

$C_{e}=(1.00 \pi)^{2}=9.87$ (For Pinned-Pinned)

$C_{e}=(1.25 \pi)^{2}=15.5$ (For Clamped-Pinned)

$C_{e}=(1.50 \pi)^{2}=22.2$ (For Clamped-Clamped) (Guo et al., 2005)

Reduced Velocity This is the velocity parameter used to determine velocity ranges at which vortex shedding induced oscillations can occur. The reduced velocity parameter, $V_{r}$, equation is:

$$
V_{r}=\frac{V_{c}}{f_{n} D}
$$

Stability Parameter: The stability parameter affects and is used to define the vortex shedding oscillations. It is defined as: 


$$
K_{s}=\frac{2 M_{e} \delta_{s}}{\rho_{w} D^{2}}
$$

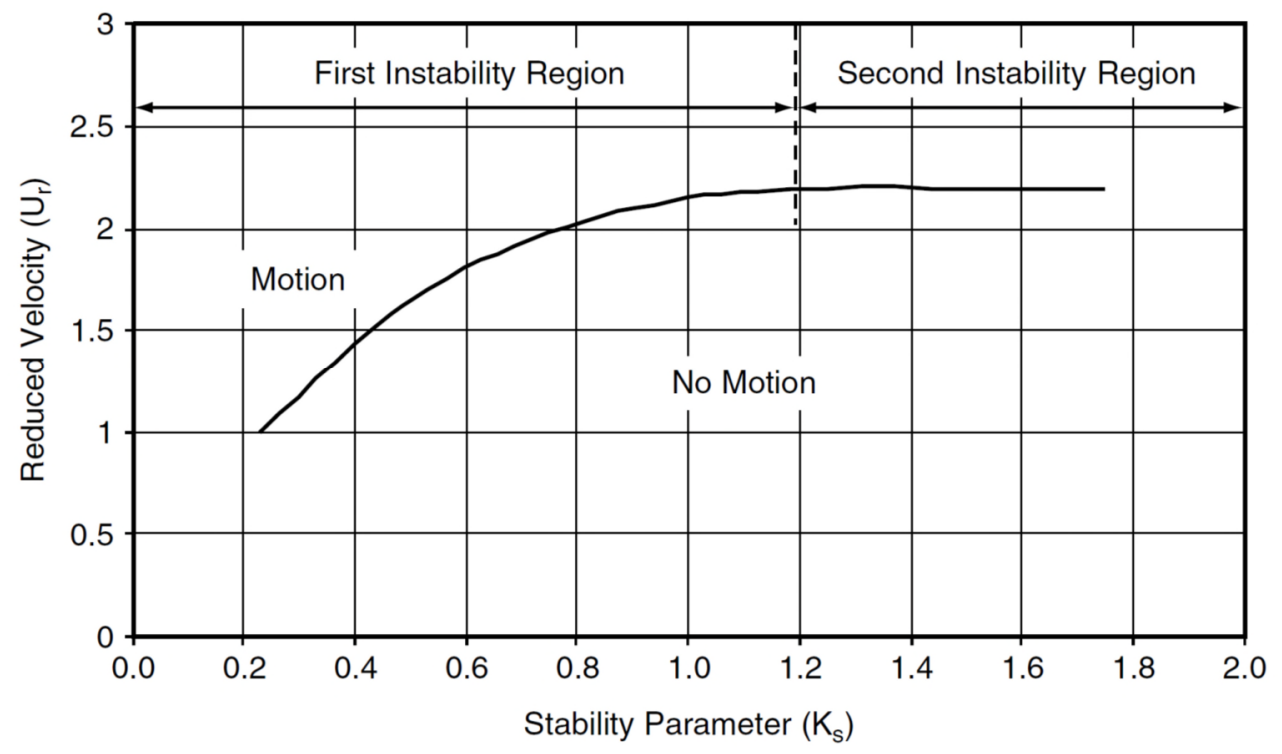

Figure 5: Reduced velocity for in-line oscillations based on the stability parameter.

Source: (Guo et al., 2005)

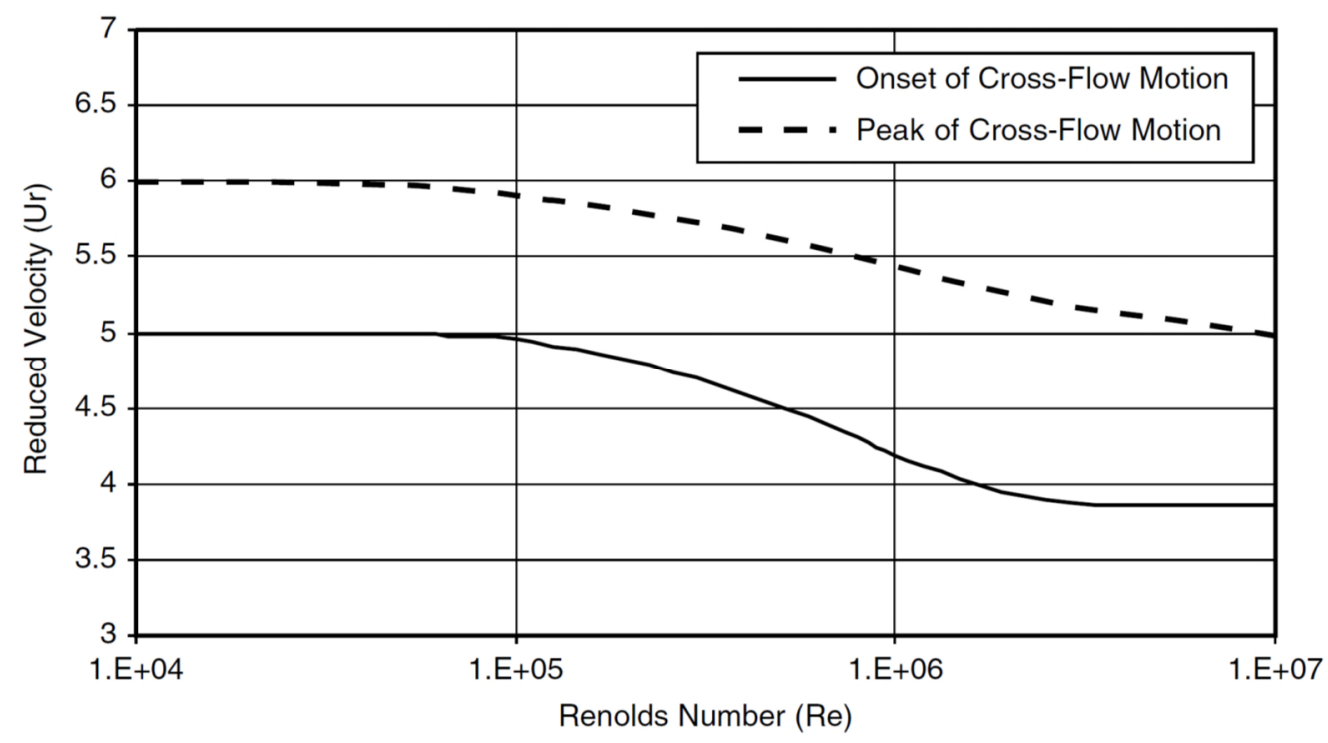

Figure 6: Reduced velocity for cross-flow oscillations based on Reynolds Number.

Source: (Guo et al., 2005)

Charts for determining Reduced Velocity for in-line and cross-flow motions 
Critical Span Length Oscillation of the pipeline may occur at the unsupported pipeline length or critical span length for a given current, based on the correlation between the reduced velocity and the natural frequency of the pipe free span.

The critical span length for cross-flow motion can be calculated from the formular:

$$
L_{c}=\sqrt{\frac{C_{e} V_{r} D_{o}}{2 \pi V_{c}} \sqrt{\frac{E I}{M_{e}}}}
$$

And for the in-line motion, the critical span length can be calculated as:

$$
L_{c}=\sqrt{\frac{C_{e}}{2 \pi f_{n}} \sqrt{\frac{E I}{M_{e}}}}
$$

Design assumptions, explanation of design procedures and further explanations on analysis of free spans are included in the Appendix.

MAFSL Calculation for 48 inches Pipeline in Hydrotest Condition

Table 3: Determination of Maximum Allowable Free Span Length (MAFSL)

Parameter Value Unit

$\begin{array}{lll}D_{0} & 1.229 & \mathrm{~m}\end{array}$

$\begin{array}{lll}D_{t} & 1.169 & \mathrm{~m}\end{array}$

$\begin{array}{lll}t_{\text {nom }} & 0.03 & \mathrm{~m}\end{array}$

$\begin{array}{lll}t_{\text {corr }} & 0.0042 & \mathrm{~m}\end{array}$

$\begin{array}{lll}t_{\text {conc }} & 0.070 & \mathrm{~m}\end{array}$

$\begin{array}{lll}h & 30 & \mathrm{~m}\end{array}$

$g \quad 9.81 \quad \mathrm{~m} / \mathrm{s}^{2}$ 


\begin{tabular}{|c|c|c|}
\hline$k$ & 0.72 & - \\
\hline$v$ & 0.3 & - \\
\hline$\mu$ & 0.6 & - \\
\hline$E$ & $210 \times 10^{9}$ & $N / m^{2}$ \\
\hline$\sigma_{y}$ & $450 \times 10^{6}$ & $N / m^{2}$ \\
\hline$\alpha$ & $1.17 \times 10^{-5}$ & $/{ }^{\circ} \mathrm{C}$ \\
\hline$P_{i}$ & $1.20 \times 10^{7}$ & $N / m^{2}$ \\
\hline$T_{o p}$ & 80 & ${ }^{\circ} \mathrm{C}$ \\
\hline$T_{a m b}$ & 10 & ${ }^{\circ} \mathrm{C}$ \\
\hline$\rho_{\text {cont }}$ & 1025 & $\mathrm{~kg} / \mathrm{m}^{3}$ \\
\hline$\rho_{s}$ & 7850 & $\mathrm{~kg} / \mathrm{m}^{3}$ \\
\hline$\rho_{\text {corr }}$ & 950 & $\mathrm{~kg} / \mathrm{m}^{3}$ \\
\hline$\rho_{\text {conc }}$ & 3040 & $\mathrm{~kg} / \mathrm{m}^{3}$ \\
\hline$\rho_{w}$ & 1025 & $\mathrm{~kg} /$ \\
\hline
\end{tabular}

Table 4: Static Analysis

\begin{tabular}{cccc}
\hline$M_{\text {cont }}$ & $\rho_{\text {cont }} A_{\text {int }}$ & $1.1 \times 10^{3}$ & $\mathrm{~kg} / \mathrm{m}$ \\
$M_{s}$ & $\rho_{s} A_{s}$ & $8.8708 \times 10^{2}$ & $\mathrm{~kg} / \mathrm{m}$ \\
$M_{\text {corr }}$ & $\rho_{\text {corr }} A_{\text {corr }}$ & $1.5295 \times 10^{1}$ & $\mathrm{~kg} / \mathrm{m}$ \\
$M_{\text {conc }}$ & $\rho_{\text {conc }} A_{\text {conc }}$ & $8.7403 \times 10^{2}$ & $\mathrm{~kg} / \mathrm{m}$
\end{tabular}




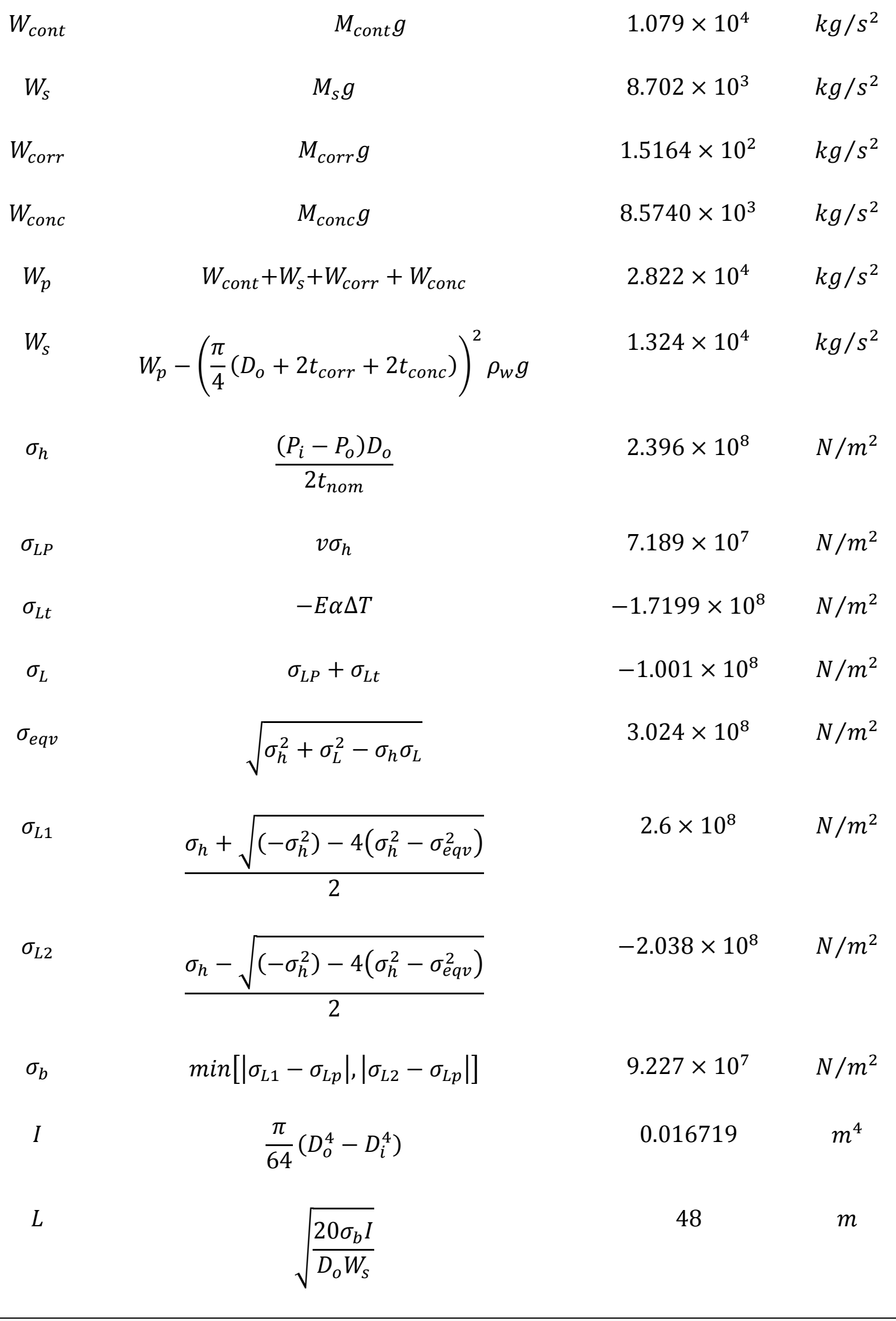


$v_{k}$

$V_{C}$

$\delta$

$C_{e}$

$D_{e}$

$C_{a}$

$M_{a}$

$M_{e}$

$K_{S}$

$R_{e}$

$V_{r} \quad$ DnV RP F-105 (Det Norske Veritas, 2006)

(Figure 5 herein)

$V_{R}$

DnV RP F-105 (Det Norske Veritas, 2006)
$1.05 \times 10^{-6} \mathrm{~m}^{2} / \mathrm{s}$

0.416

$\mathrm{m} / \mathrm{s}$

0.02

9.87

1.377

$m$

2.28

$3.482 \times 10^{3} \quad \mathrm{~kg} / \mathrm{m}$

$6.178 \times 10^{3} \mathrm{~kg} / \mathrm{m}$

0.131

$1.266 \times 10^{6}$

(Figure 6 herein)

$L_{c}$

$$
\sqrt{\frac{C_{e}}{2 \pi f_{n}} \sqrt{\frac{E I}{M_{e}}}}
$$

$m$

$L_{C}$

$$
\sqrt{\frac{C_{e} V_{r} D_{e}}{2 \pi V_{c}} \sqrt{\frac{E I}{M_{e}}}}
$$

88.4 $m$ 
Result for MAFSL

Static

$48 m$

In-line motion

$65.3 m$

Cross-flow motion

$88.4 m$

MAFSL Calculation for 48 inches Pipeline in Empty Condition

Table 6: Determination of Maximum Allowable Free Span Length (MAFSL)

\begin{tabular}{|c|c|c|}
\hline Parameter & Value & Unit \\
\hline$D_{o}$ & 1.229 & $\mathrm{~m}$ \\
\hline$D_{t}$ & 1.169 & $\mathrm{~m}$ \\
\hline$t_{\text {nom }}$ & 0.03 & $\mathrm{~m}$ \\
\hline$t_{c o r r}$ & 0.0042 & $\mathrm{~m}$ \\
\hline$t_{\text {conc }}$ & 0.070 & $\mathrm{~m}$ \\
\hline$h$ & 30 & $\mathrm{~m}$ \\
\hline$g$ & 9.81 & $\mathrm{~m} / \mathrm{s}^{2}$ \\
\hline$k$ & 0.72 & - \\
\hline$v$ & 0.3 & - \\
\hline$\mu$ & 0.6 & - \\
\hline$E$ & $210 \times 10^{9}$ & $N / m^{2}$ \\
\hline$\sigma_{y}$ & $450 \times 10^{6}$ & $N / m^{2}$ \\
\hline$\alpha$ & $1.17 \times 10^{-5}$ & $/{ }^{\circ} \mathrm{C}$ \\
\hline$P_{i}$ & 0 & $N / m^{2}$ \\
\hline
\end{tabular}




\begin{tabular}{lcc}
$T_{\text {op }}$ & 80 & ${ }^{\circ} \mathrm{C}$ \\
$T_{\text {amb }}$ & 10 & ${ }^{\circ} \mathrm{C}$ \\
$\rho_{\text {cont }}$ & 1.25 & $\mathrm{~kg} / \mathrm{m}^{3}$ \\
$\rho_{s}$ & 7850 & $\mathrm{~kg} / \mathrm{m}^{3}$ \\
$\rho_{\text {corr }}$ & & $\mathrm{kg} / \mathrm{m}^{3}$ \\
$\rho_{\text {conc }}$ & 950 & $\mathrm{~kg} / \mathrm{m}^{3}$ \\
$\rho_{w}$ & 3040 & $\mathrm{~kg}^{3}$ \\
& & \\
\hline
\end{tabular}

Table 7: Static Analysis

\begin{tabular}{lccc}
\hline$M_{\text {cont }}$ & $\rho_{\text {cont }} A_{\text {int }}$ & 1.342 & $\mathrm{~kg} / \mathrm{m}$ \\
$M_{s}$ & $\rho_{s} A_{s}$ & $8.8708 \times 10^{2}$ & $\mathrm{~kg} / \mathrm{m}$ \\
$M_{\text {corr }}$ & $\rho_{\text {corr }} A_{\text {corr }}$ & $1.5458 \times 10^{1}$ & $\mathrm{~kg} / \mathrm{m}$ \\
$M_{\text {conc }}$ & $\rho_{\text {conc }} A_{\text {conc }}$ & $8.7403 \times 10^{2}$ & $\mathrm{~kg} / \mathrm{m}$ \\
$W_{\text {cont }}$ & $M_{\text {cont }} g$ & $1.3161 \times 10^{1}$ & $\mathrm{~kg} / \mathrm{s}^{2}$ \\
$W_{s}$ & $M_{s} g$ & $8.702 \times 10^{3}$ & $\mathrm{~kg} / \mathrm{s}^{2}$ \\
$W_{\text {corr }}$ & $M_{\text {corr }} g$ & $1.5164 \times 10^{2}$ & $\mathrm{~kg} / \mathrm{s}^{2}$ \\
$W_{\text {conc }}$ & $M_{\text {conc }} g$ & $8.5740 \times 10^{3}$ & $\mathrm{~kg} / \mathrm{s}^{2}$ \\
$W_{p}$ & $W_{\text {cont }}+W_{s}+W_{\text {corr }}+W_{\text {conc }}$ & $1.744 \times 10^{4}$ & $\mathrm{~kg} / \mathrm{s}^{2}$ \\
$W_{s}$ & $W_{p}-\left(\frac{\pi}{4}\left(D_{o}+2 t_{\text {corr }}+2 t_{\text {conc }}\right)\right)^{2} \rho_{w} g$ & $2.456 \times 10^{3}$ & $\mathrm{~kg} / \mathrm{s}^{2}$
\end{tabular}




\begin{tabular}{|c|c|c|c|}
\hline$\sigma_{h}$ & $\frac{\left(P_{i}-P_{o}\right) D_{o}}{2 t_{n o m}}$ & $6.179 \times 10^{6}$ & $N / m^{2}$ \\
\hline$\sigma_{L P}$ & $v \sigma_{h}$ & $-1.854 \times 10^{6}$ & $N / m^{2}$ \\
\hline$\sigma_{L t}$ & $-E \alpha \Delta T$ & $-1.7199 \times 10^{8}$ & $N / m^{2}$ \\
\hline$\sigma_{L}$ & $\sigma_{L P}+\sigma_{L t}$ & $-1.738 \times 10^{8}$ & $N / m^{2}$ \\
\hline$\sigma_{e q v}$ & $\sqrt{\sigma_{h}^{2}+\sigma_{L}^{2}-\sigma_{h} \sigma_{L}}$ & $1.708 \times 10^{8}$ & $N / m^{2}$ \\
\hline$\sigma_{L 1}$ & $\sigma_{h}+\sqrt{\left(-\sigma_{h}^{2}\right)-4\left(\sigma_{h}^{2}-\sigma_{e q v}^{2}\right)}$ & $1.676 \times 10^{8}$ & $N / m^{2}$ \\
\hline & 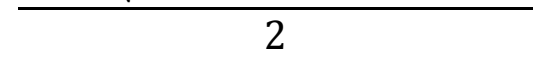 & & \\
\hline$\sigma_{L 2}$ & $\frac{\sigma_{h}-\sqrt{\left(-\sigma_{h}^{2}\right)-4\left(\sigma_{h}^{2}-\sigma_{e q v}^{2}\right)}}{2}$ & $-1.738 \times 10^{8}$ & $\mathrm{~N} / \mathrm{m}^{2}$ \\
\hline$\sigma_{b}$ & $\min \left[\left|\sigma_{L 1}-\sigma_{L p}\right|,\left|\sigma_{L 2}-\sigma_{L p}\right|\right]$ & $1.7199 \times 10^{8}$ & $N / m^{2}$ \\
\hline$I$ & $\frac{\pi}{64}\left(D_{o}^{4}-D_{i}^{4}\right)$ & 0.016719 & $m^{4}$ \\
\hline$L$ & $\sqrt{\frac{20 \sigma_{b} I}{D_{o} W_{s}}}$ & 150.9 & $m$ \\
\hline
\end{tabular}

Table 8: Dynamic Analysis

\section{Dynamic Analysis}

\begin{tabular}{ccc}
\hline$v_{k}$ & $1.05 \times 10^{-6}$ & $\mathrm{~m}^{2} / \mathrm{s}$ \\
$V_{C}$ & 0.366 & $\mathrm{~m} / \mathrm{s}$ \\
$\delta$ & 0.02 & - \\
$C_{e}$ & 9.87 & -
\end{tabular}


$D_{e}$

$C_{a}$

$M_{a}$

$M_{e}$

$K_{S}$

$R_{e}$

$V_{r}$

$V_{R}$

$L_{c}$

$L_{C}$

$$
\begin{gathered}
\sqrt{\frac{C_{e}}{2 \pi f_{n}} \sqrt{\frac{E I}{M_{e}}}} \\
\sqrt{\frac{C_{e} V_{r} D_{e}}{2 \pi V_{c}} \sqrt{\frac{E I}{M_{e}}}}
\end{gathered}
$$
herein)

6 herein)

Result for MAFSL

Static

In-line motion
$150.9 m$

$68.4 m$ 
motion

MAFSL Calculation for 48 inches Pipeline in Operating Condition

Table 9: Determination of Maximum Allowable Span Length (MAFSL)

Parameter

$D_{o}$

$D_{t}$

$t_{\text {nom }}$

$t_{\text {corr }}$

$t_{\text {conc }}$

$h$

$g$

$k$

$v$

$\mu$

E

$\sigma_{y}$

$\alpha$

$P_{i}$

$T_{o p}$

$T_{a m b}$
Value

1.229

0.03

0.0042

0.070

30

9.81

0.72

0.3

0.6

$210 \times 10^{9}$

$450 \times 10^{6}$

$1.17 \times 10^{-5}$

$9.6 \times 10^{6}$

80

10
Unit

$\mathrm{m}$

$\mathrm{m}$

$\mathrm{m}$

$\mathrm{m}$

$\mathrm{m}$

$\mathrm{m}$

$\mathrm{m} / \mathrm{s}^{2}$

$\mathrm{N} / \mathrm{m}^{2}$

$N / m^{2}$

$/{ }^{\circ} \mathrm{C}$

$N / m^{2}$

${ }^{\circ} \mathrm{C}$

${ }^{\circ} \mathrm{C}$ 
$\rho_{\text {cont }}$

$\rho_{s}$

$\rho_{\text {corr }}$

$\rho_{\text {conc }}$

$\rho_{w}$
856

7850

950

3040

1025 $\mathrm{kg} / \mathrm{m}^{3}$

$\mathrm{kg} / \mathrm{m}^{3}$

$\mathrm{kg} / \mathrm{m}^{3}$

$\mathrm{kg} / \mathrm{m}^{3}$

$\mathrm{kg} / \mathrm{m}^{3}$

Table 10: Static Analysis

\begin{tabular}{|c|c|c|c|}
\hline$M_{\text {cont }}$ & $\rho_{\text {cont }} A_{\text {int }}$ & $9.1874 \times 10^{2}$ & $\mathrm{~kg} / \mathrm{m}$ \\
\hline$M_{S}$ & $\rho_{S} A_{s}$ & $8.8708 \times 10^{2}$ & $\mathrm{~kg} / \mathrm{m}$ \\
\hline$M_{\text {corr }}$ & $\rho_{\text {corr }} A_{\text {corr }}$ & $1.5295 \times 10^{1}$ & $\mathrm{~kg} / \mathrm{m}$ \\
\hline$M_{\text {conc }}$ & $\rho_{\text {conc }} A_{\text {conc }}$ & $8.7403 \times 10^{2}$ & $\mathrm{~kg} / \mathrm{m}$ \\
\hline$W_{\text {cont }}$ & $M_{\text {cont }} g$ & $9.0128 \times 10^{3}$ & $\mathrm{~kg} / \mathrm{s}^{2}$ \\
\hline$W_{s}$ & $M_{s} g$ & $8.702 \times 10^{3}$ & $\mathrm{~kg} / \mathrm{s}^{2}$ \\
\hline$W_{\text {corr }}$ & $M_{c o r r} g$ & $1.5164 \times 10^{2}$ & $\mathrm{~kg} / \mathrm{s}^{2}$ \\
\hline$W_{\text {conc }}$ & $M_{\text {conc }} g$ & $8.5740 \times 10^{3}$ & $\mathrm{~kg} / \mathrm{s}^{2}$ \\
\hline$W_{p}$ & $W_{\text {cont }}+W_{s}+W_{\text {corr }}+W_{\text {conc }}$ & $2.644 \times 10^{4}$ & $\mathrm{~kg} / \mathrm{s}^{2}$ \\
\hline$W_{s}$ & $W_{p}-\left(\frac{\pi}{4}\left(D_{o}+2 t_{c o r r}+2 t_{\text {conc }}\right)\right)^{2} \rho_{w} g$ & $1.7199 \times 10^{4}$ & $\mathrm{~kg} / \mathrm{s}^{2}$ \\
\hline$\sigma_{h}$ & $\frac{\left(P_{i}-P_{o}\right) D_{o}}{2 t_{\text {nom }}}$ & $1.905 \times 10^{8}$ & $N / m^{2}$ \\
\hline$\sigma_{L P}$ & $v \sigma_{h}$ & $5.714 \times 10^{7}$ & $N / m^{2}$ \\
\hline
\end{tabular}




\begin{tabular}{|c|c|c|c|}
\hline$\sigma_{L t}$ & $-E \alpha \Delta T$ & $-1.7199 \times 10^{8}$ & $N / m^{2}$ \\
\hline$\sigma_{L}$ & $\sigma_{L P}+\sigma_{L t}$ & $-1.149 \times 10^{8}$ & $N / m^{2}$ \\
\hline$\sigma_{e q v}$ & $\sqrt{\sigma_{h}^{2}+\sigma_{L}^{2}-\sigma_{h} \sigma_{L}}$ & $2.671 \times 10^{8}$ & $N / m^{2}$ \\
\hline \multirow[t]{2}{*}{$\sigma_{L 1}$} & $\sigma_{h}+\sqrt{\left(-\sigma_{h}^{2}\right)-4\left(\sigma_{h}^{2}-\sigma_{e q v}^{2}\right)}$ & $2.565 \times 10^{8}$ & $N / m^{2}$ \\
\hline & 2 & & \\
\hline \multirow[t]{2}{*}{$\sigma_{L 2}$} & $\sigma_{h}-\sqrt{\left(-\sigma_{h}^{2}\right)-4\left(\sigma_{h}^{2}-\sigma_{e q v}^{2}\right)}$ & $-6.6 \times 10^{7}$ & $N / m^{2}$ \\
\hline & 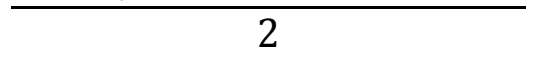 & & \\
\hline$\sigma_{b}$ & $\min \left[\left|\sigma_{L 1}-\sigma_{L p}\right|,\left|\sigma_{L 2}-\sigma_{L p}\right|\right]$ & $1.231 \times 10^{8}$ & $N / m^{2}$ \\
\hline$I$ & $\frac{\pi}{64}\left(D_{o}^{4}-D_{i}^{4}\right)$ & 0.016719 & $m^{4}$ \\
\hline$L$ & $\sqrt{\frac{20 \sigma_{b} I}{D_{o} W_{s}}}$ & 59.6 & $m$ \\
\hline
\end{tabular}

Table 11: Dynamic Analysis

\section{Dynamic Analysis}

\begin{tabular}{|c|c|c|c|}
\hline$v_{k}$ & & $1.05 \times 10^{-6}$ & $m^{2} / s$ \\
\hline$V_{C}$ & & 0.442 & $\mathrm{~m} / \mathrm{s}$ \\
\hline$\delta$ & & 0.02 & - \\
\hline$C_{e}$ & & 9.87 & - \\
\hline$D_{e}$ & $D_{o}+2 t_{\text {corr }}+2 t_{\text {conc }}$ & 1.377 & $m$ \\
\hline$C_{a}$ & $0.68+\frac{1.6}{1+5\left(\frac{e}{D_{e}}\right)}$ & 2.28 & \\
\hline
\end{tabular}




\begin{tabular}{|c|c|c|c|}
\hline$M_{a}$ & $C_{a} \rho_{w} \frac{\pi D^{2}}{4}$ & $3.482 \times 10^{3}$ & $\mathrm{~kg} / \mathrm{m}$ \\
\hline$M_{e}$ & $M_{\text {cont }}+M_{p}+M_{\text {corr }}+M_{\text {conc }}+M_{a}$ & $6.178 \times 10^{3}$ & $\mathrm{~kg} / \mathrm{m}$ \\
\hline$K_{S}$ & $\frac{2 M_{e} \delta}{\rho_{w} D_{e}^{2}}$ & 0.127 & \\
\hline$R_{e}$ & $\frac{V_{c} D}{v_{k}}$ & $4 \times 10^{5}$ & \\
\hline$V_{r}$ & $\begin{array}{l}\text { DnV RP F-105 (Det Norske Veritas, } \\
\text { 2006) (Figure } 5 \text { herein) }\end{array}$ & 1.57 & \\
\hline$V_{R}$ & DnV RP F-105 (Figure 6 herein) & 4.55 & \\
\hline$L_{c}$ & $\sqrt{\frac{C_{e}}{2 \pi f_{n}} \sqrt{\frac{E I}{M_{e}}}}$ & 63.8 & $m$ \\
\hline$L_{C}$ & $\sqrt{\frac{C_{e} V_{r} D_{e}}{2 \pi V_{c}} \sqrt{\frac{E I}{M_{e}}}}$ & 136.8 & $m$ \\
\hline
\end{tabular}

Result for MAFSL

Static

$59.608 m$

In-line motion

$63.8 m$

Cross-flow motion

$136.8 m$

\section{Recommendation}

In this work an extensive review of the different numerical and semi-empirical models were conducted. There is need to carry out more testing until adequate and reliable models is accomplished in the case of semi-empirical and CFD models for VIV prediction. On the recently updated version of the VIVANA there is still a need to enrich hydrodynamic 
coefficient database, Optimize the updated coefficient database and calibrate/ optimize the updated coefficient database against other flexible model test. Further laboratory experiment as well as field test data measurements are still needed to clarify and to support better estimation of VIV hydrodynamic forces especially for the lock-in region. Further works should also be carried out in the CFD models because they show high prospects in the prediction of IL VIV as CFD simulation of turbulent fluid flow around one or several pipes can in principle be applied for VIV assessment to overcome the inherent limitations of the state-of-practice engineering approach. The identified challenge in the prediction models for VIV is in their inability to predict pure IL displacements in the combined CF-IL mode which is of critical importance in the analysis of free spanning pipelines. Further experiments are needed in this area.

\section{Conclusion}

The pipeline span analysis in the field of hydrodynamics offshore and ocean technology is illustrated in great detail. This review shows that the span analysis for pipelines can be predicted using different analytical, semi-empirical, and numerical methods. The most dominant effect in deep water on bottom span analysis is vortex-induced vibration and this effect can be predicted best by the use of numerical methods such as VIVANA. Also, In-line and cross-flow forces can be predicted reasonably by the use of semi-empirical methods such as the VIVANA. Finally, the response amplitude and fatigue characteristics can also be predicted by the use of well-established commercial software's such as ABAQUS and ANSYS. A typical 48" offshore transportation system was designed for MAFSL using guidelines from DNV RP F-105 in order to demonstrate the procedures inherent in pipeline span analysis. The following conclusion can be drawn from the study: 
- The DNV RP F105 represents the free span assessment code and provides rational design criteria and guidance for assessment of pipeline free spans subjected to combined wave and current loading

- The tools presented herein for pipeline free span analysis include the Simulator, Pipesin, VIVSIM, SAGE Profile 3D, OFFPIPE, VIVANA, SHEAR7, FatFree and CFD based models.

- Recent developments in mathematical models were discussed such as: how the pattern of current induced drag force can be obtained using numerical model; a FEM model developed using ANSYS in order to solve fundamental equations of motion of pipe; the development of a dynamic differential equation subsea pipeline spans based on Hamilton principle; fluid structure interaction simulations of free spanning pipelines exposed to sea bottom currents was performed to better understand VIV of pipelines near seafloor; studying the excessive conservatism inherent in the DNV RP F105 using probabilistic analysis; using an approach based on nonlinear FEA to screen subsea pipeline against free spanning; and the development of PIPESOIL and SPAFAT which are based on DNV RP F105 to assess fatigue damage accumulation of evolving spans under forcing agents.

- VIVANA is a semi empirical frequency domain program based on 3D FE formulation and VIV response analysis model. The comparison between predictions by VIVANA to experimental data from a previous study shows the model gave good results for prediction of VIV response except for certain combination of modes however recent study reveals that recently updated version of VIVANA gave improved results.

- SHEAR7 is an empirical based mode superposition model which is widely used in the industry for Risers (owing to the predominantly CF VIV encountered). Previous studied revealed the SHEAR7 predicted pure IL VIV response poorly. However recently the Pure In-line VIV analysis have been incorporated. 
- Other models used for riser VIV response prediction include VIVA, VICOMO, ABAVIV, VIVIC, Deepflow, USP, Norsk Hydro, Vortex tracking, Orcaflex, ANAPIPE-VIV, RiserProd among others but are not reviewed in detail in this study because there is no study on their prediction of VIV responses due to on bottom currents with respect to Deepwater (on a free spanning subsea pipeline). There is need to test pipe-soil interaction for these models.

- Comparative study between the models used for prediction of VIV response revealed that the ABAVIV and VIVANA predicted the IL VIV responses better. However, the ABAVIV is based on the Morison's equation which is limited to shallow and intermediate water depths.

- A component of the Simulator known As the SIMLA is used to perform eigen-mode analysis. This is used in conjunction with FATFREE for pipeline free span fatigue assessment. The simulator is JP Kenny's Assessment tool which is an advanced FEA tool that allows accurate prediction of pipeline response.

- Other tools include the SPAN-CALC, MODE-CALC and the FAT-CALC which are Intecsea's assessment tools have been seamlessly integrated to facilitate an automated and quick implementation of fatigue damage assessment.

- MAFSL is a function of submerged weight, bending stress, moment of area of the pipe and outside diameter for static case while it is a function of end condition constant, stiffness, effective mass, Reynolds number, stability parameter, reduced velocity, Strouhal frequency, current velocity, effective outside diameter among others.

\section{References}

Afiinsen, K. A. (1995). Review of Free Spanning Pipelines. Proceedings of the 5th International Offshore and Polar Engineering Conference, II, 129-133.

Ai, S. M., \& Sun, L. P. (2009). The effect of functional loads on free spanning pipeline's VIV 
response. Journal of Marine Science and Application, 8(2), 151-155. https://doi.org/10.1007/s11804-009-8108-y

Alam, M. S., \& Cheng, L. (2010). A parallel three-dimensional scour model to predict flow and scour below a submarine pipeline. Central European Journal of Physics, 8(4), 604619. https://doi.org/10.2478/s11534-009-0149-y

Aristodemo, F., Tomasicchio, G. R., \& Veltri, P. (2011). New model to determine forces at onbottom slender pipelines. Coastal Engineering, 58(3), 267-280. https://doi.org/10.1016/j.coastaleng.2010.11.004

Aronsen, K. H., Larsen, C. M., \& Mork, K. (2005). Hydrodynamic coefficients from in-line VIV experiments. Proceedings of the International Conference on Offshore Mechanics and Arctic Engineering - OMAE, 1 B(Omae), 783-791. https://doi.org/10.1115/OMAE2005-67393

Bai, Y. (2001). Pipelines and Risers. Elsevier Ocean Engineering Series (Vol. 3). https://doi.org/10.1016/S1571-9952(01)80033-7

Bai, Y. (2003). Marine Structural Design (First). Elsevier.

Bai, Y., \& Bai, Q. (2005). Subsea pipelines and Risers. Elsevier.

Bai, Y., \& Bai, Q. (2010). Subsea Engineering Handbook. Elsevier. USA. https://doi.org/10.1016/B978-1-85617-689-7.10017-2

Breastrup, M. W., Anderson, J. B., Andersen, L., Bryndum, M., Christensen, C. J., \& Nelson, N. R. (2005). Design and installation of Marine Pipelines. Blakwell Science, Oxford, UK.

Brederow Shaw. (2012). Hevicote: Custom coatings and field coating services.

BSI. (2004). BS PD 8010-2 Code of Practice for Pipeline. Subsea Pipelines. British Standard Institute, (May).

Cao, X., \& Qin, Y. (2010). The Numerical Simulation of Local Scour around Offshore Pipeline. In Proceedings of the International Offshore and Polar Engineering Conference, paper No 142 (Vol. 2).

Chakrabarti, S. (2005). Handbook of Offshore Engineering. Plainfield, Illinois: Offshore Structural Analysis, Inc.

Chen, B., \& Cheng, L. (2002). Numerical Investigation of Three-dimensional Flow around a Free-Spanned Pipeline. In Proceedings of the International Offshore and Polar Engineering Conference, Paper No. 61. (Vol. 12).

Cheng, L., Yeow, K., Zhang, Z., \& Teng, B. (2009). Three-dimensional scour below offshore pipelines in steady currents. Coastal Engineering, 56(5-6), 577-590. https://doi.org/10.1016/j.coastaleng.2008.12.004

Cheng, L., Zang, Z. P., Zhao, M., \& Teng, B. (2008). Numerical Modeling of Onset Conditions of Scour below Offshore Pipeline in Steady Currents. Proceedings of the Eighteenth (2008) International Offshore and Polar Engineering Conference, Vol 2, 8, 250-254.

Cheng, L., \& Zhao, M. (2010). Numerical Model for Three-Dimensional Scour Below a Pipeline in Steady Currents. International Conference on Scour and Erosion, 2010(1990), 482-490. https://doi.org/doi:10.1061/41147(392)46

Choi, H. S. (2001). Free spanning analysis of offshore pipelines. Ocean Engineering, 28(10), 1325-1338. https://doi.org/10.1016/S0029-8018(00)00071-8 
da Silva, P. A., Temperville, A., \& Seabra Santos, F. (2006). Sand transport under combined current and wave conditions: A semi-unsteady, practical model. Coastal Engineering, 53(11), 897-913. https://doi.org/10.1016/j.coastaleng.2006.06.010

Davies, A. ., Van Rijn, L. ., Damgaard, J. ., Van de Graaff, J., \& Ribberink, J. . (2002). Intercomparison of research and practical sand transport models. Coastal Engineering, 46(1), 1-23. https://doi.org/10.1016/S0378-3839(02)00042-X

Det Norske Veritas. (2000). Environmental conditions and environmental loads. DNV RP C205.

Det Norske Veritas. (2002). Free Spanning Pipelines. Recommended Practice DNV-RP-F105.

Det Norske Veritas. (2006). Free Spanning Pipelines. Recommended Practice DNV-RP-F105, (February).

Det Norske Veritas. (2007). submarine pipeline systems. Offshore Standard DNV OS F101. Norway.

Dos Santos, M. V. F., Morooka, C. K., Caire, M., Franciss, R., \& Matt, C. G. C. (2014). A comparative study of a free span pipeline through numerical simulations. Proceedings of the International Conference on Offshore Mechanics and Arctic Engineering - OMAE, 6B, 1-9. https://doi.org/10.1115/OMAE2014-24069

Drago, M., Pigliapoco, M., \& Ciuffardi, T. (2007). Analysis of Pipeline Fatigue Damage for Scour Induced Freespans. In Proceedings of the International Offshore and Polar Engineering Conference, Paper No. 892. (pp. 892-899).

Durowoju, M. O. (2012). Vortex-induced vibration on deep water risers. Masters Thesis, Cranfield University, Bedfordshire, UK.

Eigbe, U., Fletcher, M., Hensley, M., Ling, D., \& Routh, C. (2006). Free-Span Remediation Studies for the K2 Pipe-In-Pipe Flowlines. Proceedings of Offshore Technology Conference (OTC), 1-4 May, 2006, Houston. Texas, Paper No. OTC 18312 MS., (18312MS). https://doi.org/10.4043/18312-MS

Elsayed, T., Fahmy, M., \& Samir, R. (2016). A Finite Element Model for Subsea Pipeline Stability and Free Span Screening. Canadian Journal on Mechanical Sciences and Engineering, 8 (1)(September), 1-9.

Esplin, G. D., \& Stappenbelt, B. (2011). Reducing conservatism in free spanning pipeline vortex-induced vibration fatigue analysis. Australian Journal of Mechanical Engineering, 8 (1), 11-20.

Etemad-Shahidi, A., Yasa, R., \& Kazeminezhad, M. H. (2011). Prediction of wave-induced scour depth under submarine pipelines using machine learning approach. Applied Ocean Research, 33(1), 54-59. https://doi.org/10.1016/j.apor.2010.11.002

Fard, M. G., Yeganeh-Bakhtiary, A., Cheng, L., \& Khayyer, A. (n.d.). Numerical simulation of jet flow hydrodynamics at erosion tunnel beneath offshore pipelines. Retrieved from www.manigolparvar.com/ConferencePapers/NumericalSimulationofJetFlowHydrodyna mics.Pdf.

Fyrileiv, O., \& Collberg, L. (2005). Influence of pressure in pipeline design - Effective axial force. In Proceedings of the International Conference on Offshore Mechanics and Arctic Engineering - OMAE, Vol. 3, Paper No. 629.

Gao, F., Yang, B., Yan, S., \& Wu, Y. (2006). Physical Modeling of Current-Induced Seabed Scour around a Vibrating Submarine Pipeline. In ISOPE-2006: Sixteenth(2006) 
International Offshore and Offshore and Polar Engineering Conference Proceedings, 28 May - 2 June 2006, San Fransisco, California, International Society of Offshore and Polar Engineers, USA. (pp. 108-112).

Guo, B., Song, S., Ghalambor, A., Lin, T., \& Chacko, J. (2005). Offshore Pipelines (First). USA: Gulf Professional Publishing, UK.

Huai, W., Wang, Z., Qian, Z., \& Han, Y. (2011). Numerical simulation of sandy bed erosion by 2D vertical jet. Science China Technological Sciences, 54(12), 3265-3274. https://doi.org/10.1007/s11431-011-4574-y

Huang, S., Sun, J., Abdalla, B., \& Group, W. (2017). Pipeline Span Assessment Lessons Learned from Numerical Simulations FEA Modeling for Pipeline On-Bottom Roughness Assessment. In Offshore Technology Conference, 1-4 May 2017, Houston, Texas, Offshore Technology Conference (OTC), Houston, Texas, Paper No. OTC 27846-MS (pp. 1-4). https://doi.org/10.4043/27846-MS

Huang, X. G., \& Xu, J. Q. (2010). Numerical procedure for static and dynamic analysis of fluid-conveying submarine pipeline span on linear elastic seabed. Journal of Shanghai Jiaotong University (Science), 15(6), 719-725. https://doi.org/10.1007/s12204-010-10752

Jain, R. (2012). Module on pipeline engineering.

Jiang, W., \& Lin, M. (2010). Simulating the sandwaves moving with a ultra-high resolution three dimensional hydrodynamic model. In Proceedings of the International Offshore and Polar Engineering Conference, Paper No. 233 (Vol. 1, pp. 233-238). Retrieved from http://www.scopus.com/inward/record.url?eid=2-s2.0-

77956282051\&partnerID $=40 \& m d 5=c 515 a d 895161 \mathrm{fbadb} 64 \mathrm{~d} 3 \mathrm{~b} 7 \mathrm{c} 9 \mathrm{ff} 1 \mathrm{a} 931$

Jones, N. (1995). Quasi-static analysis of structural impact damage. Journal of Constructional Steel Research, 33(3), 151-177. https://doi.org/10.1016/0143-974X(94)00002-Y

Jp, K., \& Partners. (1993). Structural analysis of pipeline spans. HSE.

Jukes, P., Eltaher, A., Wang, J., \& Duron, B. (2008). The use of advanced finite element analysis tools for the design and simulation of subsea oil and gas pipelines and components. In Petroleum Exhibition and Conference of Mexico, 11-13 November, Villahermosa, Tabasco, Mexico, PECOM, Mexico.

Jukes, P., Wang, J., \& Duron, B. (2008). Solving Pipeline Technology Challenges in the Gulf of Mexico by Innovation, Advanced Analysis Tools, and Engineering Competency. Offshore Technology Conference5-8 May, 2008, Houston, Texas, Offshore Technology Conference (OTC), Houston,Texas, Paper No. OTC 19504 MS. https://doi.org/10.4043/19504-MS

Kapuria, S., Salpekar, V. Y., \& Sengupta, S. (1999). Fatigue Due to Vortex-Induced Crossflow Oscillations in Free Spanning Pipelines Supported on Elastic Soil Bed. In Proceedings of the Ninth (1999) International Offshore and Polar Engineering Conference (Vol. II, pp. 129-203).

Kaye, D., Palmer, A., Galbraith, D., North, M., Ingram, J., Palmer, A., ... North, M. (1993). Pipeline Freespan Evaluation: A New Methodology. In Offshore Europe 93, Paper No. 253 (pp. 253-262).

Komarova, N. L., \& Hulscher, S. J. M. H. (2000). Linear instability mechanisms for sand wave formation. Journal of Fluid Mechanics, 413, 219-246. 
https://doi.org/10.1017/S0022112000008429

Komarova, N. L., \& Newell, A. C. (2000). Nonlinear dynamics of sandbanks and sand waves. Journal of Fluid Mechanics, 415(415), 285-321.

Koushan, K. (2009). Vortex induced vibrations of free span pipelines.

Larsen, C. M., Koushan, K., \& Passano, E. (2002). Frequency and time domain analysis of vortex induced vibrations for free span pipelines. In Proceedings of the International Conference on Offshore Mechanics and Arctic Engineering - OMAE, Vol. 1, Paper No. 103.

Li, Y., Lin, M., Jiang, W. B., \& Fan, F. X. (2011). Process control of the sand wave migration in Beibu Gulf of the South China Sea. Journal of Hydrodynamics, 23(4), 439-446. https://doi.org/10.1016/S1001-6058(10)60134-5

Liang, D., \& Cheng, L. (2005). Numerical modeling of flow and scour below a pipeline in currents. Part I. Flow simulation. Coastal Engineering, 52(1), 25-42. https://doi.org/10.1016/j.coastaleng.2004.09.002

Liang, D., Cheng, L., \& Li, F. (2005). Numerical modeling of flow and scour below a pipeline in currents. Part II. Scour simulation. Coastal Engineering, 52(1), 43-62. https://doi.org/10.1016/j.coastaleng.2004.09.001

Liang, D., Cheng, L., \& Yeow, K. (2005). Numerical study of the Reynolds-number dependence of two-dimensional scour beneath offshore pipelines in steady currents. Ocean Engineering, 32(13), 1590-1607. https://doi.org/10.1016/j.oceaneng.2004.10.025

Lu, L., Li, Y., \& Qin, J. (2005). Numerical simulation of the equilibrium profile of local scour around submarine pipelines based on renormalized group turbulence model. Ocean Engineering, 32(17-18), 2007-2019. https://doi.org/10.1016/j.oceaneng.2005.04.004

Martel, S. J. (2004). Mechanics of landslide initiation as a shear fracture phenomenon. Marine Geology, 203(3-4), 319-339. https://doi.org/10.1016/S0025-3227(03)00313-X

Mirmohammadi, A., \& Ketabdari, M. J. (2011). Numerical simulation of wave scouring beneath marine pipeline using smoothed particle hydrodynamics. International Journal of Sediment Research, 26(3), 331-342. https://doi.org/10.1016/S1001-6279(11)60097-8

Mørk, K. J., Fyrileiv, O., Nes, H., \& Sortland, L. (1999). A Strategy for Assessment of NonStationary Free Spans. International Conference on Offshore Mechanics and Artic Engineering, IV, 421-428.

Myrhaug, D., Ong, M. C., Føien, H., Gjengedal, C., \& Leira, B. J. (2009). Scour below pipelines and around vertical piles due to second-order random waves plus a current. Ocean Engineering, 36(8), 605-616. https://doi.org/10.1016/j.oceaneng.2009.02.007

Myrhaug, D., Ong, M. C., \& Gjengedal, C. (2008). Scour below marine pipelines in shoaling conditions for random waves. Coastal Engineering, 55(12), 1219-1223. https://doi.org/10.1016/j.coastaleng.2008.03.006

Nemeth, A. (2003). Modelling offshore sand waves. (Doctor of Philosophy thesis), Universite Twente, Netherlands.

Németh, A. A., Hulscher, S. J. M. H., \& De Vriend, H. J. (2002). Modelling sand wave migration in shallow shelf seas. Continental Shelf Research, 22(18-19), 2795-2806. https://doi.org/10.1016/S0278-4343(02)00127-9

Németh, A. A., Hulscher, S. J. M. H., \& Van Damme, R. M. J. (2006). Simulating offshore 
sand waves. Coastal Engineering, 53(2-3), 265-275. https://doi.org/10.1016/j.coastaleng.2005.10.014

Németh, A. A., Hulscher, S. J. M. H., \& Van Damme, R. M. J. (2007). Modelling offshore sand wave evolution. Continental Shelf Research, 27(5), 713-728. https://doi.org/10.1016/j.csr.2006.11.010

Nielsen, F. G., Søreide, T. H., \& Kvarme, S. O. (n.d.). VIV response of long free spanning pipelines. In Proceedings of the International Conference on Offshore Mechanics and Arctic Engineering - OMAE, Vol. 1, Paper No. 121.

Nodine, M., Gilbert, R., Kiureghian, S., Cheon, J., Wrzyszczynski, M., Coyne, M., \& Ward, E. (2007). Impact of hurricane-induced mudslides on pipelines. In 30 April - 3 May, 2007, Houston, Texas, Offshore Technology Conference (OTC), Houston, Texas, Paper No. OTC 18983-MS (Vol. 70, pp. 1-13). https://doi.org/10.4043/18983-MS

Palmer, A., C., \& King, A., R. (2004). Subsea pipeline engineering. Penwell books, Tulsa Oklahoma.

Park, H., \& Kim, C. (1997). Analytical methods for the determination of allowable free span lengths of subsea pipelines. Proceedings of the 7th International Offshore and Polar Engineering Conference, Vol. 2, Paper No. 337., 2, 337-342. Retrieved from https://www.onepetro.org/conference-paper/ISOPE-I-97-203

Passano, E., Larsen, C. M., \& Wu, J. (2010). VIV of free spanning pipelines: Comparison of response from semi-empirical code to model tests. In Proceedings of the International Conference on Offshore Mechanics and Arctic Engineering - OMAE, Vol. 6, Paper No. 567.

Pereira, A., Franco, L., Tardelli, L., Bomfimsilva, C., \& Eigbe, U. (2008). In-place free span assessment using finite element analysis. In Proceedings of the International Conference on Offshore Mechanics and Arctic Engineering - OMAE, Vol. 3, Paper No. 191.

Pontaza, J. P., Menon, R. G., Swanson, R. C., Jhingran, V., Hill, M., Kopp, F., ... Projects, S. (2010). Fluid-Structure Interaction Simulations of a Pipeline Span Exposed to Sea Bottom Currents. In Offshore Technology Conference OTC 21070. https://doi.org/10.4043/21070MS

Project consulting service inc. (1997). Analysis and assessment of unsupported subsea pipeline spans. United States department of the interior minerals management service, USA.

Raven, P. W. J., Stuart, R. J., Bray, J. A., \& Littlejohns, P. S. (1985). Full-Scale Dynamic Testing of Submarine Pipeline Spans. In Offshore Technology Conference, 6-9 May 1985, Houston, Texas, Offshore Technology Conference (OTC), Houston, Texas, Paper No. OTC 5005-MS.

Reid, A., Grytten, T. I., \& Nystrom, P. R. (2000). Case studies in pipeline free span fatigue. In Proceedings of the International Offshore and Polar Engineering Conference, Vol. 3, Paper No. 275.

Rezazadeh, K., Zhu, L., Bai, Y., \& Zhang, L. (2010). Fatigue analysis of multi-spanning subsea pipelines. In Proceedings of the International Conference on Offshore Mechanics and Arctic Engineering - OMAE, Vol. 5, Paper No. 805.

Rippon, I. J., Shah, B. C., \& White, C. N. (1986). Design and operational considerations for unsupported offshore pipeline spans. In Offshore Technology Conference (p. 18). https://doi.org/10.2118/15810-PA 
Ruby, K., \& Hartvig, P. (2008). Free-span analysis of an offshore pipeline. (M.Sc thesis), Aalborg University.

Sabag, S. R., Edge, B. L., \& Soedigdo, I. (2000). Wake II model for hydrodynamic forces on marine pipelines including waves and currents. Ocean Engineering, 27(12), 1295-1319. https://doi.org/10.1016/S0029-8018(99)00048-7

Sarpkaya, T., \& Isaacson, M. (1981). Mechanics of wave forces on offshore structures. Van nostrand Reinhold company, USA.

Shabani, M. M., Taheri, A., \& Daghigh, M. (2017). Reliability assessment of free spanning subsea pipeline. Thin-Walled Structures, 120(August), 116-123. https://doi.org/10.1016/j.tws.2017.08.026

Shear7. (2018). User Guide for SHEAR7 Version 4.10a. Document Number - t2017.j058.001. Distributed by AMOG consulting under license from MIT.

Shittu, A. A. (2012). Review of Pipeline Span Analysis and Rectification Methods. Masters Thesis, Cranfield University, Bedfordshire, UK.

Shittu, A. A., Chia, N., Garrido, J., Inkotariah, D., Nwen, J. H., Obot, M., ... Zaman, Q. (2012). Basic Design of an Offshore Transportation and Loading System.

Shittu, A. A., \& Kara, F. (2018). Review of Offshore Pipeline span creation mechanisms. International Journal of Research in Engineering and Applied Sciences, Euro-Asia Research and Development Association, 8(2), 31-53.

Smith, H. (2007). Flow and sediment dynamics aroud three dimensional structures in coastal environments. (Doctor of Philosophy thesis), Ohio State University, U. S. A.

Soedigdo, I. R., Lambrakos, K. F., \& Edge, B. L. (1998). Prediction of hydrodynamic forces on submarine pipelines using an improved Wake II Model. Ocean Engineering, 26(5), 431-462. https://doi.org/10.1016/S0029-8018(98)00006-7

Sumer, B. M., \& Fredsoe, J. (1997). Hydrodynamics around cylindrical structures. World scientific publishing, USA.

Sumer, M., \& Fredsoe, J. (2002). The mechanics of scour in the marine environment. World scientific Publishing, Singapore.

Sun, J., Jukes, P., \& Duan, G. (2009). Free span dynamics versus global buckling of the high pressure and high temperature pipeline. In Proceedings of the International Offshore and Polar Engineering Conference, Paper No. 560.

Sun, J., Jukes, P., \& Wang, J. (2011). The advancements of FEA in confronting the deepwater pipelines under high pressure and high temperature. Offshore Technology Conference, 46 October 2011, Rio de Janeiro, Brazil, Offshore Technology Conference (OTC), Houston. Texas, Paper No. OTC 22306 MS.

Tonnon, P. K., van Rijn, L. C., \& Walstra, D. J. R. (2007). The morphodynamic modelling of tidal sand waves on the shoreface. Coastal Engineering, 54(4), 279-296. https://doi.org/10.1016/j.coastaleng.2006.08.005

Tura, F., \& Vitali, L. (1991). Nonlinear Behaviour of Free Spanning Pipelines Exposed to Steady Currents:Model Tests And Numerical Simulations. International Journal of Offshore and Polar Engineering, 1(3), 184-188.

Valipour, R., Bakhtiary, A. Y., Ghaheri, A., \& Kazeminezhad, M. H. (2008). Determination of vortex shedding frequency around offshore pipeline using unsteady drag force model. In 
Proceedings of the International Conference on Offshore Mechanics and Arctic Engineering - OMAE, Vol. 5, Paper No. 613.

Van den Berg, J. (2007). Non-linear sand wave evolution. Wohrmann Print Service, Netherlands.

van den Berg, J., Sterlini, F., Hulscher, S. J. M. H., \& van Damme, R. (2012). Non-linear process based modelling of offshore sand waves. Continental Shelf Research, 37, 26-35. https://doi.org/10.1016/j.csr.2012.01.012

van Santen, R. B., de Swart, H. E., \& van Dijk, T. A. G. P. (2011). Sensitivity of tidal sand wavelength to environmental parameters: A combined data analysis and modelling approach. Continental Shelf Research, 31(9), 966-978. https://doi.org/10.1016/j.csr.2011.03.003

Wang, J., Banneyake, R., Huang, S., Jukes, P., \& Eltaher, A. (2011). The span mitigation analysis with use of advanced FEA modeling techniques. In Proceedings of the International Conference on Offshore Mechanics and Arctic Engineering - OMAE, Vol. 4, Paper No. 951.

Wang, J., Jukes, P., Wang, S., \& Duan, G. (2008). Efficient assessment of subsea pipelines and flowlines for complex spans. In Proceedings of the International Offshore and Polar Engineering Conference, Paper No. 237.

Wang, J., Xu, J., \& Jukes, P. (2010). Cost-effective span analysis methodology for different pipeline applications. In Proceedings of the International Conference on Offshore Mechanics and Arctic Engineering - OMAE, Vol. 5, Paper No. 621.

Wei, G., Lihua, C., Guangxue, L., \& Rongmin, Y. (2010). Submarine pipeline spanning mechanism on continental shelf in the South China Sea. In Proceedings of the Twentieth (2010) International Offshore and Polar Engineering Conference, 20-25 June 2010, Beijing, China, The International Society of Offshore and Polar Engineers (ISOPE), USA.

Wu, Y., \& Chiew, Y. (2011). Three dimensional scour at submarine pipelines in unidirectional steady currents. In in Burns, S., Bhatia, S., Avila, C., et al (eds.), Proceedings of the fifth international conference on scour and erosion. 7-10 November 2010, San Fransisco, American Socieof Civil Engineers (ASCE), Virginia, Paper No. GSP 210.

Xing, J. (2011). Fatigue of pipelines resting on uneven Seabed. (M.Sc thesis), Norwegian university of science and technology (NTNU), Trondheim.

Xu, J., Li, G., Horrillo, J. J., Yang, R., \& Cao, L. (2010). Calculation of maximum allowable free span length and safety assessment of the DF1-1 submarine pipeline. Journal of Ocean University of China, 9(1), 1-10. https://doi.org/10.1007/s11802-010-0001-4

Xu, W. H., Gao, X. F., \& Du, J. (2012). The prediction on in-line vortex-induced vibration of slender marine structures. Acta Mechanica Sinica/Lixue Xuebao, 28(5), 1303-1308. https://doi.org/10.1007/s10409-012-0098-3

Yang, L., Shi, B., Han, Y., Wu, J., \& Sun, X. (2010). A study of sediment motion on seabed with submarine pipeline. In Proceedings of the Twentieth (2010) International Offshore and Polar Engineering Conference, 20-25 June 2010, Beijing, China, The International Society of Offshore and Polar Engineers (ISOPE), USA.

Yeganeh-Bakhtiary, A., Kazeminezhad, M. H., Etemad-Shahidi, A., Baas, J. H., \& Cheng, L. (2011). Euler-Euler two-phase flow simulation of tunnel erosion beneath marine pipelines. Applied Ocean Research, 33(2), 137-146. 
https://doi.org/10.1016/j.apor.2011.01.001

Yeganeh Bakhtiary, A., Ghaheri, A., \& Valipour, R. (n.d.). Analysis of offshore pipeline, allowable free span length. International Journal of Civil Engineering, 5(1), 84-91.

Yin, D., Passano, E., \& Larsen, C. M. (2018). Improved in-line VIV prediction for combined in-line and cross-flow VIV responses. In Proceedings of the ASME 2017 36th international conference on ocean, offshore and arctic engineering OMAE2017-61715, Trondheim, Norway.

Zaki, A. (2006). Principles of Corrosion engineering and control (First). ButterworthHeineman, United Kingdom.

Zang, Z., Cheng, L., Zhao, M., Liang, D., \& Teng, B. (2009). A numerical model for onset of scour below offshore pipelines. Coastal Engineering, 56(4), 458-466. https://doi.org/10.1016/j.coastaleng.2008.10.001

Zhao, M., \& Cheng, L. (2010). Numerical investigation of local scour below a vibrating pipeline under steady currents. Coastal Engineering, 57(4), 397-406. https://doi.org/10.1016/j.coastaleng.2009.11.008

\section{Appendix}

\section{Design Assumptions}

Current velocity Selection: The current acting under water which has been used for the design is based on the 1 year near bottom current and 100 year near bottom current respectively. The design current velocity impact angle to the pipeline varies with pipeline route. The calculated reduced velocity, stability parameter, Reynolds number and critical span length all have to be based on a current velocity at this angle.

End Condition Selection: According to DnV RP-F105 (Det Norske Veritas, 2006), the rule of thumb for selecting the proper model for the end conditions of a pipe free span are:

- Pinned-pined: This model is used to define spans with ends free to rotate about its axis.

- Pinned-fixed: This is used for spans that fall in between pinned-pinned and fixed-fixed.

- Fixed-fixed: This condition model is used to define spans that are fixed in place by a form of support to restrict free movement 
The selection of a suitable pipe free span end condition has a contributing effect on the eigen value and frequencies of the pipeline and the allowable span length. The pipe free span end condition herein is assumed to be simply supported in a pinned-pinned end condition.

Mode shape Selection: The frequency equations and equivalent mode shape can be derived using different boundary conditions. Beam with simply supported ends: Bending moment and deflection are zero at a simply supported end. For a pipe of length $\mathrm{L}$, at length $X=0$ and $X=L$, $Y=0, Y^{\prime \prime}=0$ will be the conditions at the simply supported ends.

Pipe effective Axial Force The calculation of the pipe effective axial force is best done using non-linear finite element procedures. An assumption has been made that the pipe tension does not have significant effect on the stresses on the free span due to static loading. This has informed the recommendation of negligible tension.

\section{Design Procedures}

Certain procedures have been recommended for free span analysis. It is recommended that (Guo et al., 2005): The seabed profile should be surveyed to provide information like soil data, water depth, irregularities among others for the design. The pipeline route to be selected should have minimal free spans. The analysis is carried out for both static and dynamic conditions.

\section{Static analysis:}

- Set pipe stress limits based on recommended practice and specifications

- Calculate the maximum allowable free span length using formulars

\section{Dynamic Analysis:}

- Analyse the pipe-soil interaction to determine span reference to the seabed

- Determine the current velocity at the span reference depth. DnV RP-F105 (Det Norske Veritas, 2006) recommends that if the flow is current dominated, the free span may be assessed by adding a characteristic wave-induced flow component to the current velocity. 
- Determine the maximum allowable free span length for in-line and cross flow vortex induced vibrations (VIV) under dynamic loads.

\section{Analysis of Free Span}

According to Breastrup, et al. (Breastrup et al., 2005) the analysis of the free span requires that some considerations be made. These analyses require appropriate data in order for a proper computation to be done. These requirements are:

- Static analysis for defining the configuration of the pipeline, sectional forces and stresses under functional loads

- Eigen-value analysis for determining the modal shapes and natural frequencies

- Dynamic analysis for determining stresses under combined functional and environmental loads, pipeline deflection and sectional forces.

- Fatigue analysis for determining accumulated fatigue damage as a result of cyclic loads from vortex shedding and wave action.

The basis for free span analysis should be on static and dynamic calculations generally accepted with consideration on pipeline conditions for empty, water-filled, hydrotesting and operating pipelines (Breastrup et al., 2005).

\section{Static Analysis}

The occurrence of spans on a pipeline usually come up to two, three or more. It is recommended to analyse these spans together using the beam theory for multiple supported spans. This analysis can be complex, but it is however common to carry out the study assuming both ends of the pipe is resting on the ground and the span a single span (Jain, 2012)

The static analysis of the pipeline was carried out using a stress based method. This involves:

- Calculating the maximum bending moment

- Calculating the allowable stress

- Calculating the maximum allowable free span 
The static analysis method is used to calculate the maximum allowable free span length through the maximum bending moment $M_{M A X}$.

Xu et al (J. Xu, Li, Horrillo, Yang, \& Cao, 2010) gives the maximum bending moment equation as:

$$
\frac{W_{s} L^{2}}{12} \leq M_{M A X} \leq \frac{W_{s} L^{2}}{8}
$$

But usually taken as:

$$
\begin{gathered}
M_{M A X}=\frac{W_{s} L^{2}}{10} \\
W_{s}=m \times g=\frac{\pi}{4}\left[\left(D_{o}^{2}-D_{i}^{2}\right) \rho_{s}+\left(D_{c o r r}^{2}-D_{o}^{2}\right) \rho_{c o r r}\right. \\
+\left(D_{\text {conc }}^{2}-D_{\text {corr }}^{2}\right) \rho_{\text {conc }}+D_{i}^{2} \rho_{c o n t} \\
\left.-D_{\text {conc }}^{2} \rho_{w}\right] g
\end{gathered}
$$

Assuming marine growth is negligible

The maximum bending moment can be also expressed in terms of allowable bending stress $\sigma_{b}$ and the pipeline properties called the section modulus $z$, and the second moment of area of the pipe section $I$

$$
\begin{gathered}
M_{M A X}=z \sigma_{b} \\
M_{M A X}=\frac{2 I \sigma_{b}}{D_{o}}
\end{gathered}
$$

Where:

$$
\begin{aligned}
& z=\frac{\pi}{32}\left(\frac{D_{o}^{4}-D_{i}^{4}}{D_{o}}\right) \\
& I=\frac{\pi}{64}\left(D_{o}^{4}-D_{i}^{4}\right)
\end{aligned}
$$

Substituting equation 22 into equation 19 the maximum allowable span length $L$ will be: 


$$
L=\sqrt{\frac{20 \sigma_{b} I}{D_{o} W_{s}}}
$$

BS PD 8010 (BSI, 2004) gives the hoop stress for pipelines that satisfy the condition $\frac{D}{t}>20$ as:

$$
\sigma_{h}=\frac{\left(P_{i}-P_{o}\right) D_{o}}{2 t_{n o m}}
$$

And the poisson's effect based on the maximum hoop stress can be determined as:

$$
\sigma_{p}=-v \sigma_{h}
$$

$v=0.3$

PD 8010-2 recommends that the allowable stress should be less than the SMYS by a factor of safety $f_{d}$ which is the design factor.

$$
\sigma_{\text {all }}<f_{d} \sigma_{y}
$$

Based on this standard, a design factor of 0.72 has been used for the seabed and 0.6 for risers and landfalls. The maximum combined stress can be calculated based on the specified minimum yield strength as:

$$
\sigma_{\text {Cmax }}=f_{\text {combined }} S M Y S
$$

Also, the maximum combined stress can be calculated based on the Von mises equation as:

$$
\sigma_{\text {Cmax }}=\sqrt{\sigma_{h}^{2} \sigma_{l} \sigma_{h}+\sigma_{l}^{2}}
$$

Combining equation (29) and equation (30) gives:

$$
\sigma_{L 1, L 2}=\frac{1}{2}\left[\sigma_{h}+\sqrt{\left(-\sigma_{H}\right)^{2}-4\left(\sigma_{H}^{2}+\sigma_{C \max }^{2}\right)}\right]
$$

The longitudinal stress in tension and the longitudinal stress in compression is obtained when Poisson's effect is subtracted from both roots of $\sigma_{L}$. To determine the maximum allowable 
bending stress based on the combined stress limit, the minimum of the absolute values of the roots is determined. This gives:

$$
\sigma_{b 2}=\min \left[\left|\sigma_{L 1}-\sigma_{p}\right|,\left|\sigma_{L 2}-\sigma_{p}\right|\right]
$$

The maximum allowable bending stress can be deduced by calculating the smaller of the two based on both the longitudinal and combined stress limit

\section{Dynamic Analysis}

When spans are created on a pipeline, they have well defined natural frequencies and modes. This is because they are dynamic structures and they are subject to amplified response when they are exposed to cyclic loads that may have a frequency similar to the natural frequency. Dynamic loading: Pipelines are affected by dynamic loading which are mostly associated with the effects of environmental forces such as wave and currents forces on the pipeline surface. These forces constantly impose time varying stresses on the pipeline which can lead to the free span being overstressed beyond the allowable design stress limits or failure due to fatigue. When modelling spans, the worst environmental conditions with the probability of occurring once over a 100 year period is analysed. In the analysis of dynamic loading, the following are assumed:

- The forces on the pipeline spans are due to the sum of the effects of the wave and current induced flow around the pipeline.

- The analysis will be carried out for a pipeline span modelled as simply supported at both ends.

- The pipeline span will be considered as cylindrical because the model theories used are for cylinders in order to calculate the natural frequencies.

The various forces that act on pipelines spans due to waves and currents are inertia, drag and lift forces. The pipeline will be designed with a 30 years life and therefore data for 100 years average return period has been used. 


\section{List of Symbols}

\begin{tabular}{|c|c|}
\hline$U$ & Mean flow velocities in the $x$ direction \\
\hline$V$ & Mean flow velocities in the $y$ direction \\
\hline$x$ & The stream-wise coordinate \\
\hline$y$ & The upward-vertical coordinate \\
\hline$\rho$ & The mass density of water \\
\hline$P$ & The pressure \\
\hline$\Gamma$ & The effective viscosity \\
\hline$k$ & The turbulent kinetic energy \\
\hline$v$ & Kinematic viscosity \\
\hline$v_{t}$ & Kinematic eddy viscosity \\
\hline$P_{r}$ & Production of turbulent kinetic energy due to shear stress \\
\hline$C_{i}$ & Constant in the $k-\varepsilon$ turbulence model \\
\hline$\varepsilon$ & Dissipation of turbulent kinetic energy \\
\hline$f_{s}$ & Vortex shedding frequency or strouhal frequency \\
\hline$S$ & Strouhal number \\
\hline$V_{c}$ & Design current velocity \\
\hline$D_{o}$ & Pipe outside diameter \\
\hline$v$ & Kinematic viscosity of fluid \\
\hline$f_{n}$ & Pipe span natural frequency \\
\hline$L_{S}, L$ & Span Length \\
\hline E & Pipe's young modulus \\
\hline
\end{tabular}


$M_{e} \quad$ Effective mass of pipe

$C_{e} \quad$ End condition constant

$M_{c} \quad$ Unit mass of pipe content $($ slugs $/ f t$ Or $\mathrm{kg} / \mathrm{m})$

$M_{p} \quad$ Unit mass of pipe $\left({ }^{\text {slugs }} /{ }_{f t}\right.$ or $\left.\mathrm{kg} / \mathrm{m}\right)$

$M_{\text {corr }} \quad$ Unit mass of corrosion coating $(\operatorname{slugs} / f t$ Or $\mathrm{kg} / \mathrm{m})$

$M_{\text {conc }} \quad$ Unit mass of concrete coating $\left(\operatorname{slugs} /{ }_{f t}\right.$ Or $\left.\mathrm{kg} / \mathrm{m}\right)$

$M_{a} \quad$ Added mass $($ slugs $/ f t$ Or $\mathrm{kg} / \mathrm{m})$

$\rho_{w} \quad$ Density of the pipelines surrounding fluid

$\delta_{S} \quad$ Logarithmic decrement of structural damping

$W_{s} \quad$ Submerged weight of the pipeline per meter

$m \quad$ Mass of the pipeline per meter

$D_{o} \quad$ Outside diameter of the steel pipe

$D_{\text {corr }} \quad$ Outside diameter of the steel pipe including corrosion coating

$D_{\text {conc }} \quad$ Outside diameter of the steel pipe including corrosion and concrete coating

$\rho_{s} \quad$ Density of steel

$\rho_{\text {corr }} \quad$ Density of corrosion coating

$\rho_{\text {conc }} \quad$ Density of concrete coating

$\rho_{w} \quad$ Density of water

$g \quad$ Gravitational acceleration 


\begin{tabular}{|c|c|}
\hline$v$ & Poisson's ratio \\
\hline$f_{\text {combined }}$ & Combined stress factor \\
\hline$D_{t}$ & Internal Diameter \\
\hline$t_{\text {nom }}$ & Nominal Pipe Thickness \\
\hline$t_{\text {corr }}$ & Corrosion Coating Thickness \\
\hline$t_{\text {conc }}$ & Concrete Coating Thickness \\
\hline$h$ & Maximum Water Depth \\
\hline$k$ & Design Factor \\
\hline$\mu$ & Coefficient of friction \\
\hline E & Young's Modulus of Steel \\
\hline$\sigma_{y}$ & Specified Minimum Yield Strength \\
\hline$\alpha$ & Linear Coefficient of Expansion \\
\hline$P_{i}$ & Internal Pressure \\
\hline$T_{o p}$ & Operating Temperature \\
\hline$T_{a m b}$ & Ambient Temperature \\
\hline$\rho_{\text {cont }}$ & Density of Content \\
\hline$M_{\text {cont }}$ & Content Mass \\
\hline$M_{s}$ & Mass of steel \\
\hline$M_{\text {corr }}$ & Corrosion coating mass \\
\hline$M_{\text {conc }}$ & Concrete coating mass \\
\hline$W_{\text {cont }}$ & Content Weight \\
\hline$W_{s}$ & Weight of steel \\
\hline
\end{tabular}


$W_{\text {corr }} \quad$ Weight of corrosion coating

$W_{\text {conc }} \quad$ Weight of Concrete Coating

$W_{p} \quad$ Weight of Pipe

$W_{s} \quad$ Submerged Weight of Pipe

$\sigma_{h} \quad$ Hoop Stress

$\sigma_{L P} \quad$ Longitudinal Stress due to poissons effect

$\sigma_{L t} \quad$ Longitudinal Compressive stress due to Thermal effect

$\sigma_{L} \quad$ Longitudinal Stress

$\sigma_{e q v} \quad$ Von misses equivalent stress

$\sigma_{L 1} \quad$ Maximum Longitudinal stress by von misses criterion

$\sigma_{L 2} \quad$ Minimum Longitudinal stress by von misses criterion

$\sigma_{b} \quad$ Maximum allowable bending stress

I Moment of Area of Pipe cross section

L Maximum Allowable Free Span Length MAFSL by statics

$v_{k} \quad$ Kinematic viscosity for seawater

$C_{e} \quad$ End condition Constant

$D_{e} \quad$ Effective outside diameter

$C_{a} \quad$ Coefficient of added mass

$M_{a} \quad$ Added mass

$M_{e} \quad$ Effective mass

$K_{S} \quad$ Stability Parameter

$R_{e} \quad$ Reynolds Number 
$V_{r} \quad$ Reduced velocity for In-line motion

$V_{R} \quad$ Reduced Velocity for Cross-Flow motion

$L_{c} \quad$ Critical span length for In-line motion

$L_{C} \quad$ Critical span length for cross-flow motion

$\delta \quad$ Logarithmic decrement of structural damping

$C_{e} \quad$ End condition Constant

$M_{s} \quad$ Mass of steel 